\title{
Limit Laws in Transaction-Level Asset Price Models
}

\author{
Alexander Aue* Clifford Hurvich ${ }^{\dagger} \quad$ Philippe Soulier ${ }^{\ddagger}$
}

November 5, 2018

\begin{abstract}
We consider pure-jump transaction-level models for asset prices in continuous time, driven by point processes. In a bivariate model that admits cointegration, we allow for time deformations to account for such effects as intraday seasonal patterns in volatility, and non-trading periods that may be different for the two assets. We also allow for asymmetries (leverage effects). We obtain the asymptotic distribution of the log-price process. We also obtain the asymptotic distribution of the ordinary least-squares estimator of the cointegrating parameter based on data sampled from an equally-spaced discretization of calendar time, in the case of weak fractional cointegration. For this same case, we obtain the asymptotic distribution for a tapered estimator under more general assumptions. In the strong fractional cointegration case, we obtain the limiting distribution of a continuously-averaged tapered estimator as well as other estimators of the cointegrating parameter, and find that the rate of convergence can be affected by properties of intertrade durations. In particular, the persistence of durations (hence of volatility) can affect the degree of cointegration. We also obtain the rate of convergence of several estimators of the cointegrating parameter in the standard cointegration case. Finally, we consider the properties of the ordinary least squares estimator of the regression parameter in a spurious regression, i.e., in the absence of cointegration.
\end{abstract}

\section{Introduction}

The increasingly widespread availability of transaction-level financial price data motivates the development of models to describe such data, as well as theory for widely-used statistics

\footnotetext{
*Department of Statistics, University of California, Davis, One Shields Avenue, Davis, CA 95616, USA, email: alexaue@wald.ucdavis.edu

${ }^{\dagger}$ Stern School of Business, New York University, Henry Kaufman Management Center, 44 West Fourth Street, New York, NY 10012, USA, email: churvich@stern.nyu.edu

†Université Paris X, 200 avenue de la République, 92001 Nanterre cedex, France, email: philippe.soulier@u-paris10.fr
} 
of interest under the assumption of a given transaction-level generating mechanism. We focus here on a bivariate pure-jump model in continuous time for log prices proposed by Hurvich and Wang $(2009,2010)$ which yields fractional or standard cointegration. The motivation for using a pure-jump model is that observed price series are step functions, since no change is possible in observed prices during time periods when there are no transactions. Examples of data sets that would fit into the framework of this model include: buy prices and sell prices of a single stock; prices of two different stocks within the same industry; stock and option prices of a given company; option prices on a given stock with different degrees of maturity or moneyness; corporate bond prices at different maturities for a given company; Treasury bond prices at different maturities.

Though our paper is not entirely focused on the case of fractional cointegration, we present here some evidence that this case may arise in practice in financial econometrics. We considered option and underlying best-available bid prices for 69 different options on IBM at 390 one-minute intervals from 9:30 AM to 4 PM on May 31, 2007. Using a logperiodogram estimator based on $390^{0.5}$ frequencies, we found that the logs of the original series had estimated memory parameters close to 1 , while the residuals from the OLS regression of the log stock price on the log option price had estimated memory parameters that were typically less than 1 . Specifically, of the 69 estimated memory parameters based on these residuals, the values ranged from 0.05 to 1.14 with a mean of 0.55 and a standard deviation of 0.28 , with 30 of these estimates lying between 0.5 and 1 , while 32 were between 0 and 0.5. Thus, there is evidence for cointegration in most of the series studied, and often the evidence points towards fractional rather than standard cointegration. Furthermore, the OLS estimate of the cointegrating parameter (assuming that cointegration exists) ranged from -0.21 to 0.39 , with a mean of 0.04 and a standard deviation of 0.13 . This provides evidence that the cointegrating parameter is in general not equal to one in the present context, so it is of interest to study properties of estimates of this parameter.

Two basic questions that we address in this paper are the asymptotic distribution of the log prices as time $t \rightarrow \infty$, and of the usual OLS estimator of the cointegrating parameter based on $n$ observations of the log prices at equally-spaced time intervals as $n \rightarrow \infty$. Most of the existing methods for deriving such limit laws (see Robinson and Marinucci (2001)) cannot be applied here because the continuous-time log-price series are not diffusions and because the discretized log-price series are not linear in either an i.i.d. sequence, a martingale difference sequence or a strong mixing sequence. Nevertheless, it is of interest to know whether and under what conditions the existing limit laws, based, say, on linearity assumptions in discrete time, continue to hold under a transaction-level generating mechanism.

In the model of Hurvich and Wang (2010, 2009) the price process in continuous time is specified by a counting process giving the cumulative number of transactions up to time $t$, together with the process of changes in log price at the transaction times. This structure corresponds to the fact that most transaction-level data consists of a time stamp giving the transaction time as well as a price at that time. In such a setting, another observable 
quantity of interest is the durations, i.e., the waiting times between successive transactions of a given asset. There is a growing literature on univariate models for durations, including the seminal paper of Engle and Russell (1998) on the autoregressive conditional duration models (ACD), as well as Bauwens and Veredas (2004) on the stochastic duration model (SCD), and Deo et al. (2010) on the long-memory stochastic duration model (LMSD).

Deo et al. (2009b) showed that, subject to regularity conditions, if partial sums of centered durations, scaled by $n^{-(d+1 / 2)}$ with $d \in[0,1 / 2)$, satisfy a functional central limit theorem then the counting process $N(t)$ has long or short memory (for $d>0, d=0$, respectively) in the sense that $\operatorname{Var} N(t) \sim C t^{2 d+1}$ as $t \rightarrow \infty$ (with $C>0$ ), and they gave conditions under which this scaling would lead to long memory in volatility. In particular, LMSD durations with $d>0$ lead to long memory in volatility. The latter property has been widely observed in the econometrics literature, while evidence for long memory in durations was found in Deo et al. (2010).

Hurvich and Wang (2010, 2009) did not derive limit laws for the log price series or the OLS estimator of the cointegrating parameter, but focused instead on properties of variances and covariances for log price series and returns, and on lower bounds on the rate of convergence for the OLS estimator.

In this paper, for a slightly modified version of the model of Hurvich and Wang (2010, 2009), but under assumptions that are more general than theirs, we obtain the limit law for the log prices, and for the OLS and tapered estimators of the cointegrating parameter. In our result on the limit law for log prices, Theorem 3.1, we allow for a stochastic timevarying intensity function in the counting processes. This allows for such effects as dynamic intraday seasonality in volatility (as observed, for example, in Deo et al. (2006), as well as fixed nontrading intervals such as holidays and overnight periods. We also allow in most of our results for asymmetries (leverage effects), and show that this opens up the possibility that long memory in durations may affect the rate of convergence of estimators of the cointegrating parameter. This raises some heretofore unrecognized ambiguities in the choice of a definition of standard cointegration. Finally, we consider the properties of the ordinary least squares estimator in a spurious regression, i.e., in the absence of cointegration.

The remainder of this paper is organized as follows. In Section 2 we write the model for the log price series and state our assumptions on the counting process, the time-deformation functions, and the return shocks. In Section 3, we provide our main results on: the long-run behavior of the log-price process (Subsection 3.1), the OLS estimator for the cointegrating parameter under weak fractional, strong fractional and standard cointegration (Subsection 3.2), a tapered estimator under weak fractional, strong fractional and standard cointegration (Subsection 3.3), a continuously-averaged tapered estimator under strong fractional and standard cointegration (Subsection 3.4) and the ordinary least squares estimator in the spurious regression case (Subsection 3.5). Section 4 provides proofs. 


\section{Transaction level model}

As in Hurvich and Wang (2010, 2009), we consider a bivariate pure-jump transaction-level price model that enables cointegration. We define the log-price process $y=\left(y_{1}, y_{2}\right)=$ $(y(t): t \geq 0)$ by

$$
\begin{aligned}
& y_{1}(t)=\sum_{k=1}^{N_{1}(t)}\left(e_{1, k}+\eta_{1, k}\right)+\theta \sum_{k=1}^{N_{2}\left(t_{1, N_{1}(t)}\right)} e_{2, k}, \\
& y_{2}(t)=\sum_{k=1}^{N_{2}(t)}\left(e_{2, k}+\eta_{2, k}\right)+\theta^{-1} \sum_{k=1}^{N_{1}\left(t_{2, N_{2}(t)}\right)} e_{1, k},
\end{aligned}
$$

where for $i=1,2, N_{i}(\cdot)$ are counting processes on the real line (see Daley and Vere-Jones (2003, page 43)) such that, for $t \geq 0, N_{i}(t)$ gives the total number of transactions of Asset $i$ in $(0, t]$, and $t_{i, k}$ is the clock time (calendar time) for the $k$ th transaction of Asset $i$, with $\cdots t_{i,-1} \leq t_{i, 0} \leq 0<t_{i, 1} \leq t_{i, 2} \cdots$. The quantity $N_{2}\left(t_{1, N_{1}(t)}\right)$ denotes the number of transactions of Asset 2 between time 0 and the time $t_{1, N_{1}(t)}$ of the most recent transaction of Asset 1, with an analogous interpretation for $N_{1}\left(t_{2, N_{2}(t)}\right)$. The efficient shock sequences $\left\{e_{i, k}\right\}_{k=1}^{\infty}$ model the permanent component and the microstructure noise sequences $\left\{\eta_{i, k}\right\}_{k=1}^{\infty}$ model the transitory component of the log-price process. Efficient shock spillover effects are weighted by $\theta$ and $\theta^{-1}$, thus yielding cointegration with cointegrating parameter $\theta$, assumed nonzero. A detailed economic justification for this model, derivation of a commoncomponents representation, as well as a comparison with certain discrete-time models, is given in Hurvich and Wang (2010, 2009). more explanations

In the mathematical theory presented in this paper, all random variables and stochastic processes are defined on a single probability space $(\Omega, \mathcal{F}, \mathbb{P})$. Expectation with respect to $\mathbb{P}$ will be denoted by $\mathbb{E}$ and var and cov will denote the variance and covariance with respect to $\mathbb{P}$. Convergence in $\mathbb{P}$-probability will be denoted by $\stackrel{\mathbb{P}}{\rightarrow}$, convergence in distribution under $\mathbb{P}$ of sequences of random variables will be denoted by $\rightarrow$. We use $\Rightarrow$ to denote weak convergence under $\mathbb{P}$ in the space $\mathcal{D}([0, \infty))$ of left-limited, right-continuous (càdlàg) functions, endowed with Skorohod's $J_{1}$ topology. See Billingsley (1968) or Whitt (2002) for details about weak convergence in $\mathcal{D}([0, \infty))$. Whenever the limiting process is continuous, this topology can be replaced by the topology of uniform convergence on compact sets.

Following Daley and Vere-Jones (2003, page 47), a point process is said to be simple if the probability is zero that there exists a time $t$ at which more than one event occurs. We do not assume that the counting processes are simple. Thus we allow for the possibility that several transactions may occur at the same time. The transaction times $t_{i, k}$ are related to the point process by the following duality.

$$
N_{i}(t)=k \Leftrightarrow t_{i, k} \leq t<t_{i, k+1} .
$$

The durations are then defined by

$$
\tau_{i, k}=t_{i, k}-t_{i, k-1}
$$


If the process is simple, then $N_{i}\left(t_{i, k}\right)=k$. Otherwise, it only holds that $N_{i}\left(t_{i, k}\right) \geq k$.

There is no requirement that either the point processes $N_{i}$ or the durations $\left\{\tau_{k}\right\}$ be stationary under $\mathbb{P}$. Therefore we make the following ergodicity-type assumptions.

Assumption 2.1. The sequences $\left\{t_{i, k}\right\}$ are nondecreasing and there exists $\lambda_{i} \in(0, \infty)$ such that

$$
t_{i, k} / k \stackrel{\mathbb{P}}{\rightarrow} 1 / \lambda_{i}
$$

When the counting processes are simple, this is equivalent to $N_{i}(t) / t \stackrel{\mathbb{P}}{\rightarrow} \lambda_{i}$. Since we do not assume simplicity, we must introduce an additional assumption.

Assumption 2.2. $N_{i}(t) / t \stackrel{\mathbb{P}}{\rightarrow} \lambda_{i}$.

If the counting processes are defined from stationary ergodic durations, then Assumption 2.1 holds. If the couting processes are moreover simple, then Assumption 2.2 also holds. Conversely, if $N_{i}$ is stationary and ergodic then Assumption 2.2 holds, and if $N_{i}$ is moreover simple, then Assumption 2.1 also holds.

It should be stressed that stationarity of durations and of the point process cannot hold simultaneously under the same probability measure (except for the Poisson point process). See for instance Baccelli and Brémaud (2003) or Daley and Vere-Jones (2003) for the mathematical theory and Deo et al. (2009b) for an econometric interpretation. We now give an example which illustrates this duality.

Example 2.1. For the LMSD model, consider a probability measure (called the Palm measure) $P^{0}$ on $(\Omega, \mathcal{F})$ under which $\tau_{k}=\epsilon_{k} \mathrm{e}^{\sigma Y_{k}}$ where $\sigma$ is a positive constant, $\left\{\epsilon_{k}\right\}$ is an i.i.d. sequence of almost surely positive random variables with finite mean and $\left\{Y_{k}\right\}$ is a stationary standard Gaussian process, independent of $\left\{\epsilon_{k}\right\}$, whose covariance function goes to 0 at infinity. It follows from the latter assumption and Gaussianity that the process $\left\{Y_{k}\right\}$ is ergodic Ibragimov and Rozanov (1978), hence so is $\left\{\tau_{k}\right\}$. Suppose now that the restriction of $\mathbb{P}$ to the sigma-field generated by $N$ is equal to $P^{0}$, then Assumption 2.1 holds with $\lambda^{-1}=\mu \mathrm{e}^{\sigma^{2} / 2}$ where $\mu$ is the expectation of $\epsilon_{0}$ under $P^{0}$, and since the durations are almost surely positive, so does Assumption 2.2. Now, by the Palm duality theory, there exists a probability measure $P$ under which the associated point process $N$ is stationary (in which case, as mentioned above, the durations are no longer stationary). If the restriction of $\mathbb{P}$ to the sigma-field generated by $N$ is equal to $P$, then Assumptions 2.1 and 2.2 still hold with the same $\lambda$ as defined above.

Example 2.2. The ACD model proposed in Engle and Russell (1998) is

$$
\tau_{k}=\psi_{k} \epsilon_{k}, \quad \psi_{k}=\omega+\alpha \tau_{k-1}+\beta \psi_{k-1}, \quad k \in \mathbb{Z},
$$

where $\omega>0$ and $\alpha, \beta \geq 0,\left\{\epsilon_{k}\right\}_{k=-\infty}^{\infty}$ is an i.i.d. sequence of almost surely positive random variables with mean 1 . If $\alpha+\beta<1$ then the sequence $\left\{\tau_{k}\right\}_{k=-\infty}^{\infty}$ is strictly stationary and 
ergodic and has finite mean $\omega /(1-\alpha-\beta)$. As in the above example, we can alternatively assume that the durations form a stationary ACD process under $\mathbb{P}$ or that the associated point process is stationary under $\mathbb{P}$. In both cases, Assumptions 2.1 and 2.2 hold with $\lambda=(1-\alpha-\beta) / \omega$.

We now explain how time deformations can be used to obtain a nonstationary, possibly non simple point process from a stationary ergodic simple one, while Assumptions 2.1 and 2.2 are preserved. Let $\tilde{N}(\cdot)$ be a simple, stationary and ergodic counting process on $\mathbb{R}$ with intensity $\tilde{\lambda} \in(0, \infty)$ and let $f$ be a deterministic or random function such that $f$ is nondecreasing and has càdlàg paths with probability one. Define then

$$
N(t)=\tilde{N}(f(t))
$$

If the function $f$ is random, we assume moreover that it is independent of the counting process $\tilde{N}$.

The function $f$ is used to speed up or slow down the trading clock. To incorporate dynamic intraday seasonality in volatility, the same time deformation can be used in each trading period (of length, say, $T$ ), assuming that $f(t)$ has a periodic derivative (with period $T$ and with probability one), for example, $f(t)=t+.5 \sin (2 \pi t / T)$. Fixed nontrading intervals, say, $t \in\left[T_{1}, T_{2}\right)$, could be accommodated by taking $f(t)=f\left(T_{1}\right)$ for $t \in\left[T_{1}, T_{2}\right)$ so that $f(t)$ remains constant for $t$ in this interval, and then taking $f\left(T_{2}\right)>f\left(T_{1}\right)$ so that $f(t)$ jumps upward when trading resumes at time $T_{2}$. The jump allows for the possibility of one or more transactions at time $T_{2}$, potentially reflecting information from other markets or assets that did trade in the period $\left[T_{1}, T_{2}\right)$.

If the values of some series are only recorded at specific time points (e.g., quarterly in the case of certain macroeconomic series) this could be handled by taking the corresponding $f(t)$ to be a pure-jump function. This provides scope for considering two (or more) series, some of which are observed continuously, others at specific times, though not necessarily contemporaneously. In future work, we hope to explore this scenario in detail, and its possible connections with the MIDAS methodology, see Ghysels et al. (2006).

The use of the time-varying intensity function $f$ renders the counting process $N$ nonstationary. Since it is possible that $f$ has (upward) jumps, the $N$ may also not be simple even though the $\tilde{N}$ are simple. We now show, however, that if $\tilde{N}$ satisfies Assumptions 2.1 and 2.2, then so does the time deformed $N$ under some restrictions on $f$.

Lemma 2.1. Assume that $f$ is a nondecreasing (random) function such that $t^{-1} f(t) \stackrel{\mathbb{P}}{\rightarrow} \gamma \in$ $(0, \infty)$ and

$$
\sup _{t \geq 0}\left|f(t)-f\left(t^{-}\right)\right| \leq C
$$

with probability one, where $C \in(0, \infty)$ is a deterministic constant. Let $\tilde{N}$ be a point process such that Assumptions 2.1 and 2.2 hold for some $\lambda \in(0, \infty)$. Let $N$ be the counting process defined by $N(\cdot)=\tilde{N}(f(\cdot))$. Then Assumptions 2.1 and 2.2 hold for $N$ with $\lambda=\tilde{\lambda} \gamma$. 
Stationarity of $\tilde{N}$ is not required in this Lemma. For example, one could equally well do the time deformation on a counting process that corresponds to a stationary duration sequence (and hence the counting process is not stationary).

In order to show that our results on estimation of the cointegrating parameter (under weak fractional and standard cointegration) hold in this time deformation framework, we will in Lemma 4.7 make further assumptions on the function $f$. These assumptions mathematically embody natural economic constraints, viz. minimum duration of trading and nontrading periods, maximum duration of nontrading periods and non stoppage of trading time during trading periods.

We now state our assumptions on the return shocks.

Assumption 2.3. The efficient shocks $\left\{e_{i, k}\right\}$ are mutually independent i.i.d. sequences with zero mean and variance $\sigma_{i, e}^{2}$.

Although many of our results would continue to hold if the i.i.d. part of Assumption 2.3 were replaced by a weak-dependence assumption, we maintain the i.i.d. assumption here in keeping with the economic motivation for the model as provided by Hurvich and Wang (2010) that in the absence of the microstructure shocks and in the absence of any dependence of the efficient shocks on the counting processes, each of the log price series would be a martingale with respect to its own past. Since the trades of Asset 1 are not synchronized in calendar time or in transaction time with those of Asset 2, it seems reasonable to assume that the two efficient shock series are mutually independent, as we have done in Assumption 2.3.

The following assumption implies that the microstructure noise does not affect the limiting distribution of the log prices.

Assumption 2.4 (Microstructure Noise). The microstructure noise sequences $\left\{\eta_{i, k}\right\}$ satisfy $n^{-1 / 2} \sum_{k=1}^{[n \cdot]} \eta_{i, k} \Rightarrow 0$.

This assumption simply enforces the negligibility of the microstructure noise with respect to the long term behaviour of the log price and indeed allows us to estabish in Theorem 3.1 that the log price behaves asymptotically as a random walk (and returns at long horizons behave like a martingale difference sequence), consistent with what is assumed in most econometric literature.

Dependence between the counting processes and return shocks allows for leverage effects (for example, a correlation between a return in one time period and a squared return in a subsequent time period). A transaction-level model yielding a leverage effect was proposed (but justified only with simulations) in Hurvich and Wang (2009). Models where the point process need not be independent of the return shocks were discussed in Prigent (2001) in the context of option pricing with marked point processes.

We do not make any assumption of independence between the counting processes and the microsctucture shocks, unless explicitly noted otherwise. We will, however, assume 
that the counting processes are independent of the efficient shocks except in Theorem 3.1 and in Section 3.5.

Assumption 2.4 is all we need to assume about the microstructure noise in order to obtain a limit law for the log price series (such as Theorem 3.1 below). However, in order to discuss properties of estimators of the cointegrating parameter it is necessary to make more specific assumptions on the degree of cointegration. In Hurvich and Wang (2009, 2010), three different cases were considered, according to the strength of the memory of the microstructure noise sequences. These cases were labeled as weak fractional cointegration, strong fractional cointegration and standard cointegration. In the current context, where there may be a dependence between return shocks and counting processes, special care is needed in defining the strong fractional and standard cointegration cases, as long memory in durations may affect the rate of convergence of estimators of the cointegrating parameter in these cases. On the other hand, we will define weak fractional cointegration essentially as in Hurvich and Wang (2010).

Assumption 2.5. The shocks $\left\{e_{1, k}\right\}_{k=-\infty}^{\infty},\left\{e_{2, k}\right\}_{k=-\infty}^{\infty},\left\{\eta_{1, k}\right\}_{k=-\infty}^{\infty}$ and $\left\{\eta_{2, k}\right\}_{k=-\infty}^{\infty}$ are mutually independent.

Mutual independence of the efficient and microstructure shock series of a given asset can be justified on economic grounds, and is often made in the econometric literature for calendar-time models. See, e.g., Barndorff-Nielsen et al. (2008). Mutual independence of the two microstructure series is justified by the lack of synchronization of the trading times of the two assets.

We now discuss the weak fractional cointegration case. For $H \in(0,1)$, let $B_{H}$ denote the standard fractional Brownian motion (FBM) with Hurst index $H$, i.e. the zero mean Gaussian process with almost surely continuous sample paths and covariance function

$$
\operatorname{cov}\left(B_{H}(s), B_{H}(t)\right)=\frac{1}{2}\left\{s^{2 H}-|t-s|^{2 H}+t^{2 H}\right\} .
$$

For $H=1 / 2, B_{1 / 2}$ is the standard Brownian motion.

Assumption 2.6 (Weak Fractional Cointegration). There exists $H \in(0,1 / 2)$ such that

$$
n^{-H} \sum_{k=1}^{[n \cdot]} \eta_{i, k} \Rightarrow c_{i} B_{H}^{(i)}
$$

where $c_{1}, c_{2}$ are nonnegative constants, not both zero and $B_{H}^{(1)}$ and $B_{H}^{(2)}$ are independent standard fractional Brownian motions with common Hurst index $H$.

Assumption 2.6 is a strengthening of Assumption 2.4 and is needed to establish the asymptotic distribution of estimators of the cointegrating parameter $\theta$. Whereas Assumption 2.4 allows for cointegration, an assumption such as Assumption 2.6 is a necessary 
element for defining the degree of cointegration (the rate at which deviations from the long run cointegrating relationship disappear), and is consistent with the finding reported in Section 1 that the cointegrating residuals have weaker memory than a random walk.

Under Assumption 2.5, the independence of all the noise series implies that all the previous convergences hold jointly. The situation where one of the constants $c_{1}$ or $c_{2}$ is zero could arise naturally if the memory in one of the microstructure noise series is weaker than for the other.

In the case of weak fractional cointegration, we can define the memory parameter of the microstructure noise series as $d_{\eta}=H-1 / 2 \in(-1 / 2,0)$, and the degree of fractional cointegration (i.e. the rate of convergence of partial sums of the cointegrating error) is completely determined by $d_{\eta}$. More precisely, in this case the difference between the memory parameters of the series of log prices and the cointegrating error (observed, say, at equally-spaced intervals of calendar time) $y_{1}(j)-\theta y_{2}(j)$, is $-d_{\eta}$. This holds regardless of any dependence between the counting processes and the microstructure shocks.

Next we discuss strong fractional and standard cointegration. We start with the assumption that, for $i=1,2, \eta_{i, k}=\xi_{i, k}-\xi_{i, k-1}$ where $\left\{\xi_{i, k}\right\}$ satisfy $\sup _{k} \mathbb{E}\left[\xi_{i, k}^{2}\right]<\infty$. It then follows that the cointegrating error at time $j$ is

$$
\begin{aligned}
y_{1}(j) & -\theta y_{2}(j) \\
& =\sum_{k=N_{1}\left(t_{2, N_{2}(j)}\right)+1}^{N_{1}(j)} e_{1, k}-\theta \sum_{k=N_{2}\left(t_{1, N_{1}(j)}\right)+1}^{N_{2}(j)} e_{2, k}+\xi_{1, N_{1}(j)}-\xi_{1,0}-\theta\left(\xi_{2, N_{2}(j)}-\xi_{2,0}\right) .
\end{aligned}
$$

Under the assumptions we will make in this paper, and also under the assumptions made in Hurvich and Wang (2010, 2009), the first two terms on the righthand side of (2.5) are weakly dependent, so the degree of cointegration is determined by the rate of convergence of partial sums of $\xi_{i, N_{i}(j)}$.

Thus we will need to study the sequence $\xi_{i, N_{i}(j)}$. We do not assume that the microstructure shocks are independent of the counting processes. Thus, even if the microstructure shocks have zero mean, it may hold that $\mathbb{E}\left[\xi_{i, N_{i}(j)}\right] \neq 0$.

In view of the discussion above it is clear that in order to specify the degree of cointegration in the strong fractional and standard cointegration cases, it is necessary to make an assumption on calendar-time aggregates of $\xi_{i, N_{i}(j)}$. This is in contrast to Assumption 2.6 above where the degree of fractional cointegration is specified in terms of properties of transaction-level aggregates of the microstructure noise.

Assumption 2.7 (Strong fractional and standard cointegration). The microstucture noise sequences $\left\{\eta_{i, k}\right\}$ can be expressed as $\eta_{i, k}=\xi_{i, k}-\xi_{i, k-1}$. There exist $H \in[1 / 2,1)$, constants 
$\mu_{1}^{*}, \mu_{2}^{*}$ and nonnegative constants $c_{1}, c_{2}$, not both zero, such that

$$
n^{-H} \sum_{j=1}^{[n \cdot]}\left\{\xi_{i, N_{i}(j)}-\mu_{i}^{*}\right\} \Rightarrow c_{i} B_{H}^{(i)}
$$

where $B_{H}^{(1)}$ and $B_{H}^{(2)}$ are independent fractional Brownian motions with Hurst index $H$.

The case $H>1 / 2$ corresponds to strong fractional cointegration whereas the case $H=1 / 2$ corresponds to standard cointegration.

It might be hard to verify Assumption 2.7 unless the durations are integer valued. Since commonly-used duration models do not have integer-valued durations, we will introduce a modification of the estimators which involves integrals instead of sums, thus allowing us to avoid this restriction. This change requires a corresponding modification of Assumption 2.7.

Assumption 2.8 (Strong fractional and standard cointegration). The microstucture noise sequences $\left\{\eta_{i, k}\right\}$ can be expressed as $\eta_{i, k}=\xi_{i, k}-\xi_{i, k-1}$. There exist $H \in[1 / 2,1)$, constants $\mu_{1}^{*}, \mu_{2}^{*}$ and nonnegative constants $c_{1}, c_{2}$, not both zero, such that

$$
n^{-H} \int_{0}^{n \cdot}\left\{\xi_{i, N_{i}(s)}-\mu_{i}^{*}\right\} \mathrm{d} s \Rightarrow c_{i} B_{H}^{(i)}
$$

In their strong fractional cointegration case, Hurvich and Wang (2010) assumed, for $d_{\eta} \in(-1,-1 / 2)$, that $\operatorname{cov}\left(\xi_{i, k}, \xi_{i, k+j}\right) \sim K j^{2 d_{\xi}-1}$ as $j \rightarrow \infty$ where $K>0$ and $d_{\xi}=$ $d_{\eta}+1 \in(0,1 / 2)$. They then showed (in their Lemma 3 ), under the assumptions made there, that $\operatorname{cov}\left(\xi_{i, N_{i}(k)}, \xi_{i, N_{i}(k+j)}\right) \sim K^{\prime} j^{2 d_{\xi}-1}$ as $j \rightarrow \infty$ where $K^{\prime}>0$, so that the degree of fractional cointegration was completely determined by the rate of decay of $\operatorname{cov}\left(\xi_{i, k}, \xi_{i, k+j}\right)$. However, the proof of this result relied on the assumption that the microstructure shocks are independent of the counting processes, an assumption which we do not make here.

We next provide an example showing that under dependence between the microstructure shocks and the counting processes, it is possible for $\left\{\xi_{i, k}\right\}$ to be weakly dependent, and yet the rate of convergence of suitably normalzed integrals of the process $\left(\xi_{i, N_{i}(t)}: t \geq 0\right)$ is determined by the degree of long memory in durations. Suppressing the $i$ subscript, we have the following lemma.

Lemma 2.2. Suppose that $\left\{\tau_{k}\right\}$ is given by the LMSD model $\tau_{k}=\epsilon_{k} \mathrm{e}^{Y_{k}},\left\{\epsilon_{k}\right\}$ are i.i.d. standard exponential, independent of the stationary standard Gaussian series $\left\{Y_{k}\right\}$, which satisfies $\operatorname{cov}\left(Y_{0}, Y_{n}\right) \sim c n^{2 H_{\tau}-2}$ where $c>0$, and $H_{\tau} \in(1 / 2,3 / 4)$. Define $\xi_{k}=Y_{k+1}^{2}-1$. Then the autocovariance function of $\left\{\xi_{k}\right\}$ is summable and there exists $c^{\prime}>0$ such that

$$
n^{-1 / 2} \sum_{k=1}^{[n \cdot]} \xi_{k} \Rightarrow c^{\prime} B
$$


Nevertheless, the randomly-indexed continuous-time process $\xi_{N(t)}$ has long memory in the sense that there exists a constant $\mu^{*}$, as well as a constant $c^{\prime \prime}$, such that

$$
n^{-H_{\tau}} \int_{0}^{n .}\left\{\xi_{N(s)}-\mu^{*}\right\} \mathrm{d} s \Rightarrow c^{\prime \prime} B_{H_{\tau}} .
$$

Lemma 2.2 shows that long memory in durations can induce the same degree of long memory in the cointegrating error (2.5) in calendar-time, even though the microstructure shocks, which are the source of the cointegration, have short memory as a sequence in transaction time. In Lemma 2.2, this phenomenon was achieved by imposing a particular functional relationship between the (zero mean) microstructure noise and the persistent component of the durations, $\xi_{k}=Y_{k+1}^{2}-1$. This relationship implies a leverage effect, since $\operatorname{corr}\left(\xi_{k}, \tau_{k+1}\right)=1 / \sqrt{2(e-1)} \approx .539>0$. In other words, a strongly negative microstructure shock to the return leads to a tendency of the next observed duration, as well as subsequent durations, to be shorter than average. Such a string of short durations increases the volatility, e.g., the expectation of squared calendar-time returns, as shown, for example, under a particular return model in Deo et al. (2009b). Furthermore, evidence that stock market intertrade durations have long memory was provided in Deo et al. (2010).

In the absence of dependence between the counting processes and microstructure noise series, in both cases of strong fractional and standard cointegration, the memory of durations cannot affect the memory of the cointegrating error. See Lemma 4.9 for strong fractional cointegration and Lemma 4.10 for standard cointegration.

\section{Main results}

\subsection{The long-run behavior of the bivariate log-price process}

With the assumptions made in Section 2, the long-run behavior of the bivariate process $y=\left(y_{1}, y_{2}\right)$ can be determined. The following theorem shows that the log-prices are approximately integrated. Even though independence is assumed between the various shock series, the log-price process $y=(y(t): t \geq 0)$ exhibits a nontrivial variance-covariance structure which is determined by a complex interplay of the model parameters.

Theorem 3.1. Under Assumptions 2.1, 2.2, 2.3, 2.4, $n^{-1 / 2}\left(y_{1}(n \cdot), y_{2}(n \cdot)\right) \Rightarrow \mathbb{B}$, where

$$
\mathbb{B}=\left(\sigma_{1, e} \sqrt{\lambda_{1}} B_{1}+\theta \sigma_{2, e} \sqrt{\lambda_{2}} B_{2}, \theta^{-1} \sigma_{1, e} \sqrt{\lambda_{1}} B_{1}+\sigma_{2, e} \sqrt{\lambda_{2}} B_{2}\right) .
$$

and $B_{1}$ and $B_{2}$ are independent standard Brownian motions.

In Theorem 3.1, we have not assumed that the counting processes are independent of either the efficient shocks or the microstructure shocks. 
Hurvich and Wang $(2010,2009)$ have in their Theorem 1 computed the long-run variances of $y_{1}(t)$ and $y_{2}(t)$ which are given as $\left(\sigma_{1, e}^{2} \lambda_{1}+\theta^{2} \sigma_{2, e}^{2} \lambda_{2}\right) t$ and $\left(\sigma_{1, e}^{2} \lambda_{1} / \theta^{2}+\sigma_{2, e}^{2} \lambda_{2}\right) t$, respectively. Our theorem yields the variances as well as the covariances in the limiting distribution of $\left(t^{-1 / 2} y(t): t \geq 0\right)$. More importantly, our theorem provides the limiting distribution itself for the (normalized) log-price process $y$ which, in turn, can be used for asymptotic statistical inference. Indeed, most of the subsequent results in this paper use Theorem 3.1 and its proof as a building block. In particular, a slightly generalized version of this theorem directly yields asymptotics for estimators in spurious regressions and therefore can be used to motivate tests for the null hypothesis of no cointegration, as we describe in Section 3.5.

\subsection{OLS estimator of the cointegrating parameter}

In this section, we derive the asymptotic behavior of the ordinary least-squares estimator (OLS) of the cointegrating parameter $\theta$. To do so, we assume that the log-price series are observed at integer multiples of $\Delta t$. We will work here, without loss of generality, with $\Delta t=1$ in order to keep the notation simple. Then (2.1) and (2.2) become

$$
\begin{aligned}
& y_{1}(j)=\sum_{k=1}^{N_{1}(j)}\left(e_{1, k}+\eta_{1, k}\right)+\theta \sum_{k=1}^{N_{2}\left(t_{1, N_{1}(j)}\right)} e_{2, k}, \\
& y_{2}(j)=\sum_{k=1}^{N_{2}(j)}\left(e_{2, k}+\eta_{2, k}\right)+\theta^{-1} \sum_{k=1}^{N_{1}\left(t_{2, N_{2}(j)}\right)} e_{1, k} .
\end{aligned}
$$

Regressing $y_{1}(1), \ldots, y_{1}(n)$ on $y_{2}(1), \ldots, y_{2}(n)$ without intercept, we obtain the OLS estimator of $\theta$ as

$$
\hat{\theta}_{n}^{O L S}=\frac{\sum_{j=1}^{n} y_{2}(j) y_{1}(j)}{\sum_{j=1}^{n} y_{2}^{2}(j)} .
$$

Hurvich and Wang $(2010,2009)$ have shown in their Theorem 6 (under conditions that are

for the most part stronger than the ones we assume here) that $\hat{\theta}_{n}^{O L S}$ is weakly consistent for $\theta$ and obtained a lower bound on the rate of convergence in the case of weak fractional, strong fractional and standard cointegration. The exact limit distributions, however, were not given. We fill in this gap next for weak fractional cointegration.

\subsubsection{OLS and inference for the cointegrating parameter under weak fractional cointegration}

Theorem 3.2. Let Assumptions 2.1, 2.2, 2.3, 2.5 and 2.6 hold. Assume in addition that the counting processes $N_{1}$ and $N_{2}$ are mutually independent and independent of the efficient shocks and there exists a constant $C$ such that

$$
\sup _{s \geq 0} \mathbb{E}\left[t_{i, N_{i}(s)+1}-s\right] \leq C \text {. }
$$


Then

$$
n^{1 / 2-H}\left(\hat{\theta}_{n}^{\text {OLS }}-\theta\right) \rightarrow \Sigma_{1} \frac{\int_{0}^{1} B(t) B_{H}(t) \mathrm{d} t}{\int_{0}^{1} B^{2}(t) \mathrm{d} t}
$$

where $B$ is a standard Brownian motion, $B_{H}$ is a fractional Brownian motion, independent of $B$, and

$$
\Sigma_{1}^{2}=\frac{c_{1}^{2} \lambda_{1}^{2 H}+\theta^{2} c_{2}^{2} \lambda_{2}^{2 H}}{\theta^{-2} \lambda_{1} \sigma_{1, e}^{2}+\lambda_{2} \sigma_{2, e}^{2}}
$$

The result in Theorem 3.2 is similar to that obtained in Robinson and Marinucci (2001, Proposition 6.5, formula (6.8)), under their Assumption 6.1, for which a sufficient condition (their formula (6.5)) was verified in Marinucci and Robinson (2000) to hold for weak (but not strong) fractional cointegration in the case where the process is linear with respect to i.i.d. innovations.

Next, we provide sufficient conditions for Condition (3.3) to hold for the LMSD and ACD models under both frameworks for stationarity considered in Examples 2.1 and 2.2.

- Note first that as long as the point processes $N_{i}$ are stationary under $\mathbb{P}$, then condition (3.3) holds provided that $\mathbb{E}\left[t_{1}\right]<\infty$ (equivalently $E^{0}\left[\tau_{0}^{2}\right]<\infty$ where $E^{0}$ represents expectation with respect to the Palm measure), since the forward recurrence time $\left\{t_{i, N_{i}(s)}-s\right\}$ is then stationary.

- If the durations form a stationary LMSD sequence under $\mathbb{P}$, then Lemma 4.8 shows that if $\mathbb{E}\left[\epsilon_{0}^{q}\right]<\infty$ for all $q \geq 1$, then (3.3) holds.

- If the durations form a stationary ACD sequence under $\mathbb{P}$, then Lemma 4.6 shows that (3.3) holds as long as $\mathbb{E}\left[\tau_{0}^{3}\right]<\infty$. By Carrasco and Chen (2002, Corollary 6), this holds true if $\mathbb{E}\left[\left(\beta+\alpha \epsilon_{t}\right)^{3}\right]<1$.

- If $\tilde{N}$ is a point process that satisfies Condition (3.3) under $\mathbb{P}$, and if $f$ is a time deformation function that satisfies the economically justified assumptions of Lemma 4.7, then the time deformed point process $N$ (defined by $N(t)=\tilde{N}(f(t))$ still satisfies (3.3).

Next, we consider inference for the cointegrating parameter $\theta$ under weak fractional cointegration. It is seen from Theorem 3.2 that the asymptotic distribution of $n^{1 / 2-H}\left(\hat{\theta}_{n}^{O L S}\right.$ $\theta$ ) depends on a variety of unknown quantities. To alleviate the dependence on nuisance parameters and to thereby facilitate inference on $\theta$, we consider the $t$-statistic for testing the null hypothesis $H_{0}: \theta=\theta_{0}$. If the null hypothesis is true, then the $t$-statistic is given by

$$
t_{n}=\frac{\hat{\theta}_{n}^{O L S}-\theta}{\hat{\sigma}_{\hat{\theta}_{n}^{O L S}}}
$$


where $\hat{\sigma}_{\hat{\theta}_{n}^{O L S}}$ is the traditional estimated standard error for $\hat{\theta}_{n}^{O L S}$, with

$$
\hat{\sigma}_{\hat{\theta}_{n}^{O L S}}^{2}=\frac{n^{-1} \sum_{j=1}^{n}\left[y_{1}(j)-\hat{\theta}_{n}^{O L S} y_{2}(j)\right]^{2}}{\sum_{j=1}^{n} y_{2}^{2}(j)} .
$$

We have the following theorem.

Theorem 3.3. Under the assumptions of Theorem 3.2,

$$
n^{-1 / 2} t_{n} \rightarrow\left[\frac{\int_{0}^{1} B^{2}(t) d t \int_{0}^{1} B_{H}^{2}(t) d t}{\left[\int_{0}^{1} B(t) B_{H}(t) d t\right]^{2}}-1\right]^{-1 / 2}
$$

where $B$ is a standard Brownian motion and $B_{H}$ is a standard fractional Browninan motion, independent of $B$.

Note that by the Cauchy-Schwarz Inequality,

$$
\left|\int_{0}^{1} B(t) B_{H}(t) d t\right|^{2} \leq \int_{0}^{1} B^{2}(t) d t \int_{0}^{1} B_{H}^{2}(t) d t
$$

and in fact the inequality above is strict since $B$ and $B_{H}$ are mutually independent, so that the limiting distribution in Theorem 3.3 is well-defined.

Note that the limiting distribution in Theorem 3.3 depends only on $H$. Thus, if $H$ can be consistently estimated, one can conduct asymptotically valid hypothesis tests for $\theta$ based on the test statistic $n^{-1 / 2} t_{n}$. The asymptotic null distribution of the test statistic is given by Theorem 3.3, and it is easily seen that if $\theta \neq \theta_{0}$ then $n^{-1 / 2} t_{n}$ diverges.

We now show that $H$ can indeed be consistently estimated, using an aggregation method considered in Giraitis et al. (1999). Let $m$ be an integer sequence such that $1 / m+m / n \rightarrow 0$. We work with the differences of the cointegrating residuals, $x_{j}=\Delta\left(y_{1}(j)-\hat{\theta} y_{2}(j)\right)$, where $\Delta$ is the differencing operator. Divide the data set into contiguous, non-overlapping blocks of size $m$. The $k$ th block average is

$$
X_{k}^{(m)}=\frac{1}{m} \sum_{t=1}^{m} x_{t+(k-1) m} .
$$

Denote the sample variance of these block averages by

$$
s_{m}^{2}=\lfloor n / m\rfloor^{-1} \sum_{k=1}^{\lfloor n / m\rfloor}\left(X_{k}^{(m)}\right)^{2} .
$$

Now, define $\hat{H}=1+\frac{\log s_{m}^{2}}{2 \log m}$. The next theorem states that $\hat{H}$ is a consistent estimator of $H$ under assumptions that imply those of Theorem 3.2, but are more restrictive. We make these restrictions to facilitate a reasonably simple proof. 
Theorem 3.4. Let Assumptions 2.3 and 2.5 hold. Assume that $\eta_{i, t}=\sigma_{i}\left\{B_{i, H}(t)-B_{i, H}(t-\right.$ $1)\}, i=1,2$ where $B_{i, H}$ are mutually independent standard fractional Brownian motions with $H \in(0,1 / 2)$. Assume that $N_{1}$ and $N_{2}$ are stationary mutually independent ergodic point processes, independent of the processes $B_{i, H}$, such that $\mathbb{E}\left[N_{i}^{4}(1)\right]<\infty$ and

$$
\sup _{t \geq 2} \mathbb{E}\left[\left(\frac{N_{i}(t)}{t}\right)^{4 H-8} \mathbf{1}_{\left\{N_{i}(t)>0\right\}}\right]<\infty .
$$

Then, $\hat{H} \stackrel{\mathbb{P}}{\rightarrow} H$.

We now continue the discussion of the LMSD and ACD models (see Example 2.1 and 2.2 and the discussion immediately after Theorem 3.2). The proof of the claims is in Section 4.5, following Lemma 4.11 which it uses.

- Assume that the point process $N$ is stationary under the probability $\mathbb{P}$ and that under the Palm probability $P^{0}$ the durations form an LMSD sequence $\left\{\tau_{k}\right\}$ with memory parameter $H_{\tau} \in(1 / 2,1)$ as in Example 2.1. If $E^{0}\left[\epsilon_{0}^{9-4 H}\right]<\infty$ condition (3.4) holds. If $E^{0}\left[\epsilon_{0}^{q}\right]<\infty$ with $q>4 /\left(1-H_{\tau}\right)$, then $\mathbb{E}\left[N^{4}(1)\right]<\infty$.

- Assume that point process $N$ is stationary under the probability $\mathbb{P}$ and that under the Palm probability $P^{0}$ the durations form an ACD sequence $\left\{\tau_{k}\right\}$ as in Example 2.2. If moreover $\epsilon_{0}$ admits a strictly positive density on $[0, \infty)$ and $E^{0}\left[\tau_{1}^{9-4 H+\eta}\right]<\infty$ for some $\eta>0$, then $\mathbb{E}\left[N^{4}(1)\right]<\infty$ and condition (3.4) holds. A condition for $E^{0}\left[\tau_{1}^{9-4 H+\eta}\right]<\infty$ is $E^{0}\left[\left(\alpha \epsilon_{0}+\beta\right)^{9-4 H+\eta}\right]<1$.

\subsubsection{OLS under strong fractional and standard cointegration}

We now consider the case where the microstructure noise series $\left\{\eta_{i, k}\right\}$ are differences of strongly or weakly dependent processes $\left\{\xi_{i, k}\right\}$.

Theorem 3.5. Let Assumptions 2.1, 2.2, 2.3, 2.5, and 2.7 hold. Assume moreover that

- the efficient shocks are i.i.d. Gaussian,

- the counting processes $N_{1}$ and $N_{2}$ are independent of each other and independent of the microstructure noise sequences and of the efficient shocks and there exists a constant $C$ such that

$$
\sup _{t \geq 0} \mathbb{E}\left[\left(t_{i, N_{i}(t)+1}-t\right)^{2}\right] \leq C .
$$

- $\mathbb{E}\left[\xi_{i, k}\right]=0, \sup _{k} \mathbb{E}\left[\xi_{i, k}^{2}\right]<\infty$, and $\xi_{i, 0}=0$.

Then, 
- if $1 / 2<H<1$,

$$
n^{3 / 2-H}\left(\hat{\theta}_{n}^{O L S}-\theta\right) \rightarrow \sqrt{\frac{c_{1}^{2}+\theta^{2} c_{2}^{2}}{\theta^{-2} \lambda_{1} \sigma_{1, e}^{2}+\lambda_{2} \sigma_{2, e}^{2}}} \frac{\int_{0}^{1} B(s) \mathrm{d} B_{H}(s)}{\int_{0}^{1} B^{2}(s) \mathrm{d} s},
$$

where $B$ is standard Brownian motion independent of the standard fractional Brownian motion $B_{H}$;

- if $H=1 / 2, n\left(\hat{\theta}_{n}^{O L S}-\theta\right)=O_{P}(1)$.

The rate of convergence obtained in the standard cointegration case improves on the one obtained by Hurvich and Wang (2010). The sufficient conditions for (3.3) discussed after Theorem 3.2 become sufficient conditions for (3.5) after augmenting by 1 the exponent in the moment conditions appearing there. The assumptions in Theorem 3.5 are quite strong, ruling out leverage effects and providing one motivation for our subsequent consideration of tapered estimators.

\subsection{A Tapered Estimator of the Cointegrating parameter}

Even in existing discrete-time models for cointegration the OLS estimator lacks any particular optimality properties. Here we consider an estimator based on discrete Fourier transforms of the tapered differences of $y_{1}(j), y_{2}(j), 1 \leq j \leq n$. It was shown in Chen and Hurvich (2003a) that this estimator can have a faster rate of convergence than OLS in certain cases of fractional cointegration. In the weak fractional cointegration case, our limit results for the tapered estimator (Theorem 3.6) are obtained under identical conditions as those assumed in Theorem 3.2 for OLS. However, under strong fractional and standard cointegration, the conditions for our results on the tapered estimator (Theorem 3.7) allow for leverage, unlike the corresponding theorem for OLS.

We introduce all relevant notation using a generic time series $\left\{x_{j}\right\}_{j=-\infty}^{\infty}$. Let $h: I \mapsto \mathbb{R}$ be a general continuous taper function on an open interval $I$ containing $[0,1]$ such that $h(0)=h(1)=0$. For $\ell=1,2, \ldots$, denote by $\omega_{\ell}=2 \pi \ell / n$ the Fourier frequencies. The tapered DFT of $\left\{x_{j}\right\}_{j=-\infty}^{\infty}$ with taper function $h$ is defined by

$$
d_{x, \ell}=\sum_{j=1}^{n} h\left(\frac{j}{n}\right) x_{j} \mathrm{e}^{\mathrm{i} j \omega_{\ell}}=\sum_{j=1}^{n} h_{\ell}\left(\frac{j}{n}\right) x_{j} .
$$

where $h_{\ell}(t)=h(t) \mathrm{e}^{2 \pi i \ell t}$. Denote by $\left\{\Delta x_{j}\right\}_{j=-\infty}^{\infty}$ the first difference of the series $\left\{x_{j}\right\}$, where $\Delta x_{j}=x_{j}-x_{j-1}$. We define the tapered DFT of the first difference $\left\{\Delta x_{j}\right\}_{j=-\infty}^{\infty}$ with taper function $h$ by

$$
d_{\Delta x, \ell}=\sum_{j=1}^{n} h\left(\frac{j}{n}\right) \Delta x_{j} \mathrm{e}^{\mathrm{i} j \omega_{\ell}}=\sum_{j=1}^{n} h_{\ell}\left(\frac{j}{n}\right) \Delta x_{j} .
$$


In our setting, we observe the cointegrated component processes $y_{1}$ and $y_{2}$ at equidistant sample points. Defining the cointegrating error $z_{j}=y_{1, j}-\theta y_{2, j}$ and following Chen and Hurvich (2003b), we can now introduce the estimator

$$
\hat{\theta}_{n}^{T a p}=\operatorname{Re}\left(\tilde{\theta}_{n}\right)
$$

where $\operatorname{Re}(z)$ signifies the real part of a complex number $z=a+\mathrm{i} b$ and, letting $\bar{z}=a-\mathrm{i} b$ be the complex conjugate of $z$,

$$
\tilde{\theta}_{n}=\frac{\sum_{\ell=1}^{m} d_{\Delta y_{1}, \ell} \bar{d}_{\Delta y_{2}, \ell}}{\sum_{\ell=1}^{m}\left|d_{\Delta y_{2}, \ell}\right|^{2}}
$$

Therein, any tapered DFT of differenced sequences is defined according to (3.7). Note that $\hat{\theta}_{n}^{\text {Tap }}$ is the real part of the ratio of the averaged tapered cross-periodogram between the series $y_{1}$ and $y_{2}$ and the averaged tapered periodogram of the series $y_{2}$.

\subsubsection{Discrete tapered estimator under weak fractional cointegration}

Theorem 3.6. Let Assumptions 2.1, 2.2, 2.3, 2.5 and 2.6 hold. Assume moreover that the counting processes are mutually independent and independent of the efficient shocks and (3.3) holds. Then

$$
n^{1 / 2-H}\left(\hat{\theta}_{n}^{\text {Tap }}-\theta\right) \rightarrow \sqrt{\frac{c_{1}^{2} \lambda_{1}^{2 H}+\theta^{2} c_{2}^{2} \lambda_{2}^{2 H}}{\theta^{-2} \lambda_{1} \sigma_{1, e}^{2} \lambda_{1}+\lambda_{2} \sigma_{2, e}^{2}}} \frac{\sum_{\ell=1}^{m} \operatorname{Re}\left(\int_{0}^{1} h_{\ell}(s) \mathrm{d} B(s) \int_{0}^{1} h_{\ell}(t) \mathrm{d} B_{H}(t)\right)}{\sum_{\ell=1}^{m}\left|\int_{0}^{1} h_{\ell}(s) \mathrm{d} B(s)\right|^{2}}
$$

where $B$ is a standard Brownian motion, $B_{H}$ is a standard fractional Brownian motion and $B$ and $B_{H}$ are independent.

Since the assumptions of Theorem 3.6 are the same as in Theorem 3.2, the comments immediately following that theorem also applies here.

\subsubsection{Discrete tapered estimator under strong fractional and standard coin- tegration}

Theorem 3.7. Let Assumptions 2.1, 2.2, 2.3 2.5 and 2.7 hold. Assume morover that the counting processes are mutually independent and independent of the efficient shocks and (3.5) holds.

- If $1 / 2<H<1$, then

$$
n^{3 / 2-H}\left(\hat{\theta}_{n}^{T a p}-\theta\right) \rightarrow \sqrt{\frac{c_{1}^{2}+\theta^{2} c_{2}^{2}}{\theta^{-2} \lambda_{1} \sigma_{1, e}^{2}+\lambda_{2} \sigma_{2, e}^{2}}} \frac{\sum_{\ell=1}^{m} \operatorname{Re}\left(\int_{0}^{1} h_{\ell}(s) \mathrm{d} B(s) \int_{0}^{1} h_{\ell}^{\prime}(s) \mathrm{d} B_{H}(s)\right)}{\sum_{\ell=1}^{m}\left|\int_{0}^{1} h_{\ell}(s) \mathrm{d} B(s)\right|^{2}}
$$

where $B_{H}$ is a standard fractional Brownian motion independent of the standard Brownian motion $B$. 
- If $H=1 / 2, n\left(\hat{\theta}_{n}^{T a p}-\theta\right)=O_{P}(1)$.

The assumptions of this theorem are weaker than those of Theorem 3.5 on the OLS estimator. The microstructure shocks are not assumed to be independent of the counting processes and the efficient shocks are not assumed to be Gaussian. Theorem 3.5 can presumably be proved without the Gaussian assumption. It might be much more difficult in the proof of Theorem 3.5 to avoid the assumption of independence between the microstructure shocks and the counting processes.

\subsection{A Continuous-Time Tapered Estimator}

The estimators of $\theta$ we have considered so far are based on equally-spaced observations of the log price series. However, under the model (2.1), (2.2), a continuous-time record is available, and it is of interest to consider using all of the available data to estimate $\theta$. Here, for the sake of theoretical tractability, we consider a tapered estimator $\tilde{\theta}$ based on continuously-averaged $\log$ prices on adjacent non-overlapping time intervals. Since the problems with discretization appear only in the strong fractional and standard cointegration cases, we only consider them in this section. There is no difference in the case of weak fractional cointegration.

We first establish some notation. Let $\{X(t)\}$ be any time series defined for all $t \geq 0$, and suppose that we have data on $\left\{X_{t}\right\}$ for $t \in[0, T]$. Let $\delta>0$ be fixed. In practice, we might take $\delta$ to be 5 minutes, but the choice of $\delta$ does not affect the asymptotic distribution we derive below. Define $n=\lfloor T / \delta\rfloor, \tilde{X}(0)=0$, and

$$
\tilde{X}(k)=\int_{u=(k-1) \delta}^{k \delta} X(u) \mathrm{d} u, k=1, \cdots, n .
$$

Then we can define an estimator $\tilde{\theta}_{\delta}$ based on these averaged observations by

$$
\hat{\theta}_{n, \delta}^{T a p}=\operatorname{Re}\left(\tilde{\theta}_{n, \delta}\right)
$$

with

$$
\tilde{\theta}_{n, \delta}=\frac{\sum_{\ell=1}^{m} d_{\Delta \tilde{y}_{1}, \ell} \bar{d}_{\Delta \tilde{y}_{2}, \ell}}{\sum_{\ell=1}^{m}\left|d_{\Delta \tilde{y}_{2}, \ell}\right|^{2}}
$$

\subsubsection{Continuous-time tapered estimator under strong fractional and stan- dard cointegration}

Theorem 3.8. Let Assumptions 2.1, 2.2, 2.3, 2.5 and 2.8 hold. Assume morover that the counting processes are mutually independent and independent of the efficient shocks and (3.5) holds. 
- If $1 / 2<H<1$, then

$$
n^{3 / 2-H}\left(\hat{\theta}_{n, \delta}^{T a p}-\theta\right) \rightarrow \sqrt{\frac{\delta^{2 H}\left(c_{1}^{2}+\theta^{2} c_{2}^{2}\right)}{\theta^{-2} \lambda_{1} \sigma_{1, e}^{2}+\lambda_{2} \sigma_{2, e}^{2}}} \frac{\sum_{\ell=1}^{m} \operatorname{Re}\left(\int_{0}^{1} h_{\ell}(s) \mathrm{d} B(s) \int_{0}^{1} h_{\ell}^{\prime}(s) \mathrm{d} B_{H}(s)\right)}{\sum_{\ell=1}^{m}\left|\int_{0}^{1} h_{\ell}(s) \mathrm{d} B(s)\right|^{2}}
$$

where $B_{H}$ is a standard FBM independent of the standard Brownian motion B.

- If $H=1 / 2, n\left(\hat{\theta}_{n, \delta}^{T a p}-\theta\right)=O_{P}(1)$.

Because Assumption 2.8 involves an integral rather than a sum, we are able to verify that it holds for certain models with noninteger durations such as ACD and LMSD under certain relationships with the microstructure shocks.

In Theorem 3.8, we allow for leverage effects, and therefore care is required in defining standard cointegration. As demonstrated in Lemma 2.2 (which assumes LMSD durations) if there is a leverage effect, even when the microstructure shocks are the differences of a weakly-dependent sequence, the cointegrating error need not be $I(0)$. In such a case we have strong fractional cointegration rather than the standard cointegration which might have been anticipated.

It is also possible that even though a leverage effect exists, the memory of durations has no effect on the degree of cointegration. Specifically, if in Lemma 2.2 we replace $\xi_{k}=Y_{k+1}^{2}-1$ by $\xi_{k}=H_{2}\left(Y_{k+1}\right)-.75 H_{3}\left(Y_{k+1}\right)$, where $H_{2}(y)=y^{2}-1$ and $H_{3}(y)=y^{3}-3 y$ (the second and third Hermite polynomials, respectively), then there is a leverage effect with $\operatorname{corr}\left(\tau_{k+1}, \xi_{k}\right)=.082$. Nevertheless it follows from an argument similar to the proof of Lemma 2.2 that Assumption 2.8 holds in this example with $H=1 / 2$, so that we have standard cointegration and Theorem 3.8 holds with $H=1 / 2$.

Lemma 4.10 provides an example of standard cointegration allowing for both time deformation and dependence between the counting processes and microstructure shocks. Theorem 3.8 would hold for this example with $H=1 / 2$.

\subsection{Spurious Regressions}

In this subsection only, we consider a non-cointegrated version of the model defined by (2.1) and (2.2),

$$
\begin{aligned}
& y_{1}(t)=\sum_{k=1}^{N_{1}(t)}\left(e_{1, k}+\eta_{1, k}\right)+\theta_{21} \sum_{k=1}^{N_{2}\left(t_{1, N_{1}(t)}\right)} e_{2, k}, \\
& y_{2}(t)=\sum_{k=1}^{N_{2}(t)}\left(e_{2, k}+\eta_{2, k}\right)+\theta_{12} \sum_{k=1}^{N_{1}\left(t_{2, N_{2}(t)}\right)} e_{1, k},
\end{aligned}
$$

where $\theta_{12} \neq \theta_{21}^{-1}$. We examine here the properties of the OLS estimator in the (spurious) regression of $y_{1}$ on $y_{2}$ in discrete time and then briefly discuss corresponding tests for the 
null hypothesis of cointegration. Corollary 3.1 below follows directly from the proof of Theorem 3.1.

Corollary 3.1. If Assumptions 2.1, 2.2, 2.3 and 2.4 are satisfied and $y=\left(y_{1}, y_{2}\right)$ is given by (3.8) and (3.9) with $\theta_{12} \neq \theta_{21}^{-1}$, then as $n \rightarrow \infty$,

$$
\left(\frac{1}{\sqrt{n}} y(n u): u \in[0,1]\right) \Rightarrow B_{y}=\left(B_{y}(u): u \in[0,1]\right),
$$

where $B_{y}$ is a bivariate Brownian motion with $2 \times 2$ covariance matrix $\Sigma=\left(\Sigma_{i, j}: i, j=1,2\right)$ given by the entries

$$
\begin{gathered}
\Sigma_{1,1}=\lambda_{1} \sigma_{1, e}^{2}+\theta_{21}^{2} \lambda_{2} \sigma_{2, e}^{2}, \quad \Sigma_{2,2}=\theta_{12}^{2} \lambda_{1} \sigma_{1, e}^{2}+\lambda_{2} \sigma_{2, e}^{2} \\
\Sigma_{1,2}=\theta_{12} \lambda_{1} \sigma_{1, e}^{2}+\theta_{21} \lambda_{2} \sigma_{2, e}^{2}=\Sigma_{2,1} .
\end{gathered}
$$

Next, we consider the discretization of $y_{1}(t)$ and $y_{2}(t)$ given by (3.8) and (3.9) at integer time values,

$$
\begin{aligned}
& y_{1, j}=\sum_{k=1}^{N_{1}(j)}\left(e_{1, k}+\eta_{1, k}\right)+\theta_{21} \sum_{k=1}^{N_{2}\left(t_{1, N_{1}(j)}\right)} e_{2, k}, \\
& y_{2, j}=\sum_{k=1}^{N_{2}(j)}\left(e_{2, k}+\eta_{2, k}\right)+\theta_{12} \sum_{k=1}^{N_{1}\left(t_{2, N_{2}(j)}\right)} e_{1, k} .
\end{aligned}
$$

Regressing $y_{1,1}, \ldots, y_{1, n}$ on $y_{2,1}, \ldots, y_{2, n}$ without intercept, we obtain the OLS estimator

$$
\hat{\delta}_{n}=\frac{\sum_{j=1}^{n} y_{2, j} y_{1, j}}{\sum_{j=1}^{n} y_{2, j}^{2}} .
$$

Corollary 3.2 below follows directly from Corollary 3.1 and the Continuous Mapping Theorem.

Corollary 3.2. If Assumptions 2.1, 2.2, 2.3 and 2.4 are satisfied and $y=\left(y_{1}, y_{2}\right)$ is given by (3.8) and (3.9) with $\theta_{12} \neq \theta_{21}^{-1}$, then as $n \rightarrow \infty$,

$$
\hat{\delta}_{n} \rightarrow \frac{\int_{0}^{1} B_{2, y}(u) B_{1, y}(u) d u}{\int_{0}^{1} B_{1, y}^{2}(u) d u}
$$

where $B_{y}=\left(B_{1, y}, B_{2, y}\right)$ is the bivariate Brownian motion given in Corollary 3.1.

Corollary 3.2 together with Corollary 3.1 can be used to motivate tests for the null hypothesis of no cointegration. We do not pursue the details here, but it seems clear that the null distribution for unit root tests based on the residuals $\left\{y_{1, j}-\hat{\delta}_{n} y_{2, j}\right\}_{j=1}^{n}$ can be derived from Corollaries 3.1 and 3.2, and that these null distributions will have form similar to the distributions listed, for example, in Hamilton (1994, Proposition 19.4). 


\section{Proofs}

Proof of Lemma 2.1. Write

$$
\frac{N(t)}{t}=\frac{\tilde{N}(f(t))}{t}=\frac{\tilde{N}(f(t))}{f(t)} \frac{f(t)}{t} .
$$

By assumption, $f(t) \mapsto \infty$ (in probability if $f$ is random), thus $\tilde{N}(f(t)) / f(t)$ converges in probability to $\tilde{\lambda}$. By assumption, it also holds that $t^{-1} f(t) \stackrel{\mathbb{P}}{\rightarrow} \gamma$. Thus, $\frac{N(t)}{t} \stackrel{\mathbb{P}}{\rightarrow} \tilde{\lambda} \gamma$, so that Assumption 2.2 holds for $N$ with $\lambda=\tilde{\lambda} \gamma$. Next, we note that $N\left(t_{k}^{-}\right) \leq k$, thus

$$
\begin{aligned}
1 & \leq \frac{N\left(t_{k}\right)}{k}=1+\frac{N\left(t_{k}\right)-k}{k} \leq 1+\frac{N\left(t_{k}\right)-N\left(t_{k}^{-}\right)}{k} \\
& =1+\frac{\tilde{N}\left(f\left(t_{k}^{-}\right), f\left(t_{k}\right)\right]}{k} \leq 1+\frac{\tilde{N}\left(f\left(t_{k}\right)-C, f\left(t_{k}\right)\right]}{f\left(t_{k}\right)} \frac{f\left(t_{k}\right)}{k}
\end{aligned}
$$

using the definition of $N$ and the boundedness requirement on the jumps of $f$. Since $f\left(t_{k}\right)$ tends to infinity, it suffices to prove that if Assumptions 2.1 and 2.1 hold for $\tilde{N}$, then for any fixed positive $C$, it holds that

$$
\frac{\tilde{N}(t-C, t]}{t} \stackrel{\mathbb{P}}{\rightarrow} 0
$$

Without loss of generality, set $\tilde{\lambda}=1$. Fix some $\epsilon \in(0,1 / 2)$. Since $\tilde{t}_{k} / k \stackrel{\mathbb{P}}{\rightarrow} 1$, with probability arbitrarily close to 1 (say bigger than $1-\epsilon$ ), there exists $k_{0}$ such that $\tilde{t}_{k} / k \in$ $[1-\epsilon, 1+\epsilon]$ for all $k \geq k_{0}$. For $k \geq k_{0}$, if $t_{k} \in(t-C, t]$, it necessarily holds that $t-C \leq k(1+\epsilon)$ and $k(1-\epsilon) \leq t$. Hence, $(t-C) /(1+\epsilon) \leq k \leq t /(1-\epsilon)$. This implies that for $t$ large enough $\left(t<C+k_{0}(1+\epsilon)\right), \tilde{N}(t-C, t)$ is less than the number of integers between $(t-C) /(1+\epsilon)$ and $t /(1-\epsilon)$, i.e. $\tilde{N}(t-C, t] \leq c \epsilon t$, for some constant $c>0$. Thus $\tilde{N}(t) / t \leq c \epsilon$ with probability tending to 1 , and this proves that $\tilde{N}(t-C, t] / t \stackrel{\mathbb{P}}{\rightarrow} 0$. It follows that $N\left(t_{k}\right) / k=1+o_{P}(1)$, i.e. $N\left(t_{k}\right) / t_{k}$ converges in probability to 1 . Thus

$$
\frac{t_{k}}{k}=\frac{t_{k}}{N\left(t_{k}\right)} \frac{N\left(t_{k}\right)}{k} \stackrel{\mathbb{P}}{\rightarrow} \frac{1}{\lambda} .
$$

Proof of Lemma 2.2. Denote $H_{\tau}$ by $H$ to simplify the notation. Define $\xi_{k}=Y_{k+1}^{2}-$ 1. Then $\xi_{k}$ is centered, has finite variance summable autocovariance function, since $\operatorname{cov}\left(\xi_{0}, \xi_{k}\right)=2 \operatorname{cov}^{2}\left(Y_{0}, Y_{k+1}\right)$. Thus $\left\{\xi_{k}\right\}$ has a summable autocovariance function because $H \in(1 / 2,3 / 4)$. By Arcones (1994, Theorem 4), this implies that $\left\{\xi_{k}\right\}$ is in the domain of attraction of the standard Brownian motion, i.e.

$$
n^{-1 / 2} \sum_{k=1}^{[n \cdot]} \xi_{k} \Rightarrow c^{\prime} B
$$


with $c^{\prime 2}=\operatorname{var}\left(\xi_{0}\right)+2 \sum_{k=1}^{\infty} \operatorname{cov}\left(\xi_{0}, \xi_{k}\right)$. This proves $(2.6)$.

Assume now that $\tau_{k}=\epsilon_{k} \mathrm{e}^{\sigma Y_{k}}$ (with $\sigma=1$ in the statement of the Lemma). The properties of Hermite polynomials yield that $\mathbb{E}\left[\mathrm{e}^{\sigma Y_{0}} H_{j}\left(Y_{0}\right)\right]=\sigma^{j} \mathrm{e}^{\sigma^{2} / 2}$ for all $j \geq 1$. Denote now $\lambda^{-1}=\mathbb{E}\left[\tau_{k}\right]=\mathbb{E}\left[\mathrm{e}^{\sigma Y_{k}}\right]=\mathrm{e}^{\sigma^{2} / 2}, m=\mathbb{E}\left[\xi_{k-1} \tau_{k}\right]=\mathbb{E}\left[\left(Y_{k}^{2}-1\right) \mathrm{e}^{Y_{k}}\right]=\sigma^{2} \mathrm{e}^{\sigma^{2} / 2}$ and $G(y)=\left(y^{2}-1\right) \mathrm{e}^{\sigma y}-m$. We now prove that (2.7) holds with $\mu^{*}=\lambda m$. Write

$$
\begin{aligned}
\int_{0}^{T} & \left(\xi_{N(s)}-\lambda m\right) \mathrm{d} s \\
& =t_{1} \xi_{0}+\sum_{k=1}^{N(T)} \tau_{k+1} \xi_{k}-\lambda m T+\left(t_{N(T)+1}-T\right) \xi_{N(T)+1} \\
& =t_{1} \xi_{0}+\sum_{k=1}^{N(T)}\left(\epsilon_{k+1}-1\right) \xi_{k} \mathrm{e}^{\sigma Y_{k+1}}+\sum_{k=0}^{N(T)} G\left(Y_{k+1}\right) \\
& +m(N(T)-\lambda T)-\left(t_{N(T)+1}-T\right) \xi_{N(T)+1}
\end{aligned}
$$

By Lemma 4.8 and applying Hölder's inequality, it can be shown that $\left(t_{N(T)+1}-T\right)\left(\xi_{N(T)+1}-\right.$ $\rho)=O_{P}(1)$. Since the sequence $\left\{\epsilon_{k}\right\}$ is independent of the Gaussian process $\left\{Y_{k}\right\}$, the second term in the righthand side of (4.1) is in the domain of attraction of the standard Brownian motion, and the normalizing sequence is $\sqrt{n}$. Thus we must obtain the joint asymptotic behaviour of $\sum_{k=1}^{N(T t)} G\left(Y_{k}\right)$ and $N(T t)-\lambda T t$.

The durations are in the domain of attraction of the fractional Brownian motion with Hurst index $H$, since

$$
\sum_{k=1}^{n}\left(\tau_{k}-\lambda^{-1}\right)=\sum_{k=1}^{n}\left(\epsilon_{k}-1\right) \mathrm{e}^{\sigma Y_{k}}+\sum_{k=1}^{n}\left(\mathrm{e}^{\sigma Y_{k}}-\lambda^{-1}\right) .
$$

The first term in the righthand side is $O_{P}(\sqrt{n})$ and the second sum, suitably normalized converges to the fractional Brownian motion with Hurst index $H$ because the function $x \mapsto \mathrm{e}^{\sigma x}-\lambda^{-1}$ has Hermite rank 1. See e.g. Arcones (1994). More precisely, let $c_{1}=$ $\mathbb{E}\left[Y_{1} \mathrm{e}^{\sigma Y_{1}}\right]=\sigma \mathrm{e}^{\sigma^{2} / 2}$ and define $g(y)=\mathrm{e}^{\sigma y}-\lambda^{-1}-c_{1} y$. The function $g$ has Hermite rank 2, and since $H \in(1 / 2,3 / 4)$, this implies that

$$
\operatorname{var}\left(\sum_{k=1}^{n} g\left(Y_{k}\right)\right)=O(n)
$$

Thus $\sum_{k=1}^{n}\left(\tau_{k}-\lambda^{-1}\right)$ is asymptotically equivalent to $c_{1} \sum_{k=1}^{n} Y_{k}$. Let $B_{H}$ denote the standard fractional Brownian motion with hurst index $H$. The assumption on the covariance of the Gaussian process $\left\{y_{k}\right\}$ implies that

$$
n^{-H} \sum_{k=1}^{[n \cdot]} Y_{k} \Rightarrow \varphi B_{H}
$$


with $\varphi^{2}=c /\{H(2 H-1)\}$. Denote now $c_{2}=\mathbb{E}\left[Y_{1} G\left(Y_{1}\right)\right]=\sigma\left(\sigma^{2}+2\right) \mathrm{e}^{\sigma^{2} / 2}$ and define $h(y)=G(y)-c_{2} y$. Then $h$ has Hermite rank 2 and thus by similar arguments as above, $\sum_{k=1}^{n} G\left(Y_{k}\right)$ is asymptotically equivalent to $c_{2} \sum_{k=1}^{n} Y_{k}$. Thus we obtain

$$
n^{-H}\left(\sum_{k=1}^{[n t]}\left(\tau_{k}-\lambda^{-1}\right), \sum_{k=1}^{[n t]} G\left(Y_{k}\right)\right) \Rightarrow\left(c_{1} \varphi B_{H}(t), c_{2} \varphi B_{H}(t)\right) .
$$

By Vervaat's Lemma (see Vervaat (1972) or Resnick (2007, Proposition 3.3)), the previous convergence implies that

$$
n^{-H}\left(N(n t)-\lambda n t, \sum_{k=1}^{[n t]} G\left(Y_{k}\right)\right) \Rightarrow\left(-\lambda c_{1} \varphi B_{H}(\lambda t), c_{2} \varphi B_{H}(t)\right) .
$$

By the continuity of the composition map, this yields

$$
n^{-H}\left(N(n t)-\lambda n t, \sum_{k=1}^{N(n t)} G\left(Y_{k}\right)\right) \Rightarrow\left(-\lambda c_{1} \varphi B_{H}(\lambda t), c_{2} \varphi B_{H}(\lambda t)\right) .
$$

Next we obtain that

$$
n^{-H}\left\{\sum_{k=1}^{N(n t)} G\left(Y_{k}\right)+m(N(n t)-\lambda n t)\right\} \Rightarrow \varphi\left(c_{2}-\lambda m c_{1}\right) B_{H}(\lambda t)
$$

with $c_{2}-\lambda m c_{1}=2 \sigma \mathrm{e}^{\sigma^{2} / 2}>0$. We conclude that $n^{-H} \int_{0}^{n \cdot}\left\{\xi_{N(s)}-\lambda m\right\} \mathrm{d} s \Rightarrow \varphi\left(c_{2}-\lambda m c_{1}\right) B_{H}$.

\subsection{Proof of Theorem 3.1 and Corollary 3.1}

We first need the following Lemma.

Lemma 4.1. Under Assumption 2.1 and 2.2, $N_{i}\left(t_{j, N_{j}(n t)}\right) / n$ converges in probability uniformly on compact sets to $\lambda_{i} t$, where $\{i, j\}=\{1,2\}$.

Proof of Lemma 4.1. The sequence of (random) functions $N_{i}(n \cdot) / n$ is nondecreasing and converges pointwise in probability to $\lambda_{i} t$ by ergodicity. A sequence of nondecreasing function converging to a continuous function converges uniformly on compact sets. This results is known as Dini's Theorem. Cf. Resnick (1987, page 3). Thus the convergence of $N_{i}(n \cdot) / n$

is uniform on compact sets. Assumptions 2.1 and 2.2 imply that $N_{i}(t) \stackrel{\mathbb{P}}{\rightarrow} \infty$ and $t_{i, n} \stackrel{\mathbb{P}}{\rightarrow} \infty$. Thus

$$
\frac{N_{i}\left(t_{j, N_{j}(n u)}\right)}{n}=\frac{N_{i}\left(t_{j, N_{j}(n u)}\right)}{t_{j, N_{j}(n u)}} \times \frac{t_{j, N_{j}(n u)}}{N_{j}(n u)} \times \frac{N_{j}(n u)}{n} \stackrel{\mathbb{P}}{\rightarrow} \lambda_{i} \times \frac{1}{\lambda_{j}} \times \lambda_{j} u=\lambda_{i} u .
$$

Applying again Dini's lemma, we also have that $N_{i}\left(t_{j, N_{j}(n u)}\right) / n$ converges uniformly on compact sets to $\lambda_{i} u$. 
Proof of Theorem 3.1. Denote $S_{i, n}^{e}(t)=\sum_{k=1}^{[n t]} e_{i, k}$ and $S_{i, n}^{\eta}(t)=\sum_{k=1}^{[n t]} \eta_{i, k}, i=1,2$. Under Assumptions 2.3 and $2.4, n^{-1 / 2}\left(S_{1, n}^{e}, S_{2, n}^{e}, S_{1, n}^{\eta}, S_{1, n}^{\eta}\right)$ converges weakly to $\left(\sigma_{1, e} B_{1}, \sigma_{2, e} B_{2}, 0,0\right)$, where $B_{1}$ and $B_{2}$ are independent standard Brownian motions. This follows from the independence of $e_{1}$ and $e_{2}$ and the local uniform convergence to 0 in probability of $n^{-1 / 2} S_{i, n}^{\eta}$. With the previous notation, (3.8) and (3.9) become

$$
\begin{aligned}
& y_{1}(n t)=S_{1, n}^{e}\left(N_{1}(n t)\right)+\theta_{21} S_{2, n}^{e}\left(N_{2}\left(t_{1, N_{1}(n t)}\right)\right)+S_{1, n}^{\eta}\left(N_{1}(n t)\right), \\
& y_{2}(n t)=S_{2, n}^{e}\left(N_{2}(n t)\right)+\theta_{12} S_{1, n}^{e}\left(N_{1}\left(t_{2, N_{2}(n t)}\right)\right)+S_{2, n}^{\eta}\left(N_{2}(n t)\right) .
\end{aligned}
$$

By Lemma 4.1 and the continuity of the composition map on $\mathcal{C} \times \mathcal{C}$ endowed with the metric of uniform convergence on compact sets (see e.g. Billingsley (1968, Chapter 3, Section 17)), we obtain the joint convergence of

$$
\begin{aligned}
n^{-1 / 2}\left(S_{1, n}^{e}\left(N_{1}(n \cdot)\right), S_{1, n}^{e}\left(N_{1}\left(t_{2, N_{2}(n \cdot)}\right)\right),\right. & \\
& \left.S_{2, n}^{e}\left(N_{2}(n \cdot)\right), S_{2, n}^{e}\left(N_{2}\left(t_{1, N_{1}(n \cdot)}\right)\right), S_{1, n}^{\eta}\left(N_{1}(n \cdot)\right), S_{2, n}^{\eta}\left(N_{2}(n \cdot)\right)\right)
\end{aligned}
$$

to $\left(\sigma_{1, e} \sqrt{\lambda_{1}} B_{1}, \sigma_{1, e} \sqrt{\lambda_{2}} B_{1}, \sigma_{2, e} \sqrt{\lambda_{2}} B_{2}, \sigma_{2, e} \sqrt{\lambda_{2}} B_{2}, 0,0\right)$. This yields Corollary 3.1 and Theorem 3.1 by setting $\theta_{21}=\theta$ and $\theta_{12}=\theta^{-1}$.

\subsection{Proof of Theorems 3.23 .33 .4 and 3.5}

Proof of Theorem 3.2. Write

$$
\hat{\theta}_{n}^{O L S}=\theta+\frac{\sum_{j=1}^{n}\left\{y_{1}(j)-\theta y_{2}(j)\right\} y_{2}(j)}{\sum_{j=1}^{n} y_{2}^{2}(j)} .
$$

Assumptions 2.1, 2.2, 2.3, 2.5 and 2.6 imply those of Theorem 3.1. Thus we can apply the Continuous Mapping Theorem and obtain

$$
n^{-2} \sum_{j=1}^{n} y_{2}^{2}(j) \rightarrow\left\{\theta^{-2} \lambda_{1} \sigma_{1, e}^{2}+\lambda_{2} \sigma_{2, e}^{2}\right\} \int_{0}^{1} B^{2}(s) \mathrm{d} s,
$$

where $B$ is a standard Brownian motion. Thus, in order to study the convergence of $\hat{\theta}_{n}^{O L S}-\theta$ suitably renormalized, it suffices to study the sum

$$
\sum_{j=1}^{n}\left\{y_{1}(j)-\theta y_{2}(j)\right\} y_{2}(j)
$$


We further decompose the cointegrating error. Denote

$$
\begin{aligned}
y_{1}^{e}(j) & =\sum_{k=1}^{N_{1}(j)} e_{1, k}+\theta \sum_{k=1}^{N_{2}\left(t_{1, N_{1}(j)}\right)} e_{2, k}, \quad y_{1}^{\eta}(j)=\sum_{k=1}^{N_{1}\left(t_{2, N_{2}(j)}\right)} \eta_{1, k}, \\
y_{2}^{e}(j) & =\sum_{k=1}^{N_{2}(j)} e_{2, k}+\theta^{-1} \sum_{k=1}^{N_{1}(j)} e_{1, k}, \quad y_{2}^{\eta}(j)=\sum_{k=1}^{N_{2}(j)} \eta_{2, k}, \\
r_{1, j} & =\sum_{k=N_{1}\left(t_{2, N_{2}(j)}\right)+1}^{N_{1}(j)} e_{1, k}, \quad r_{2, j}=\sum_{k=N_{2}\left(t_{1, N_{1}(j)}\right)+1}^{N_{2}(j)} e_{2, k} .
\end{aligned}
$$

With this notation, we can write

$$
\sum_{j=1}^{n}\left\{y_{1}(j)-\theta y_{2}(j)\right\} y_{2}(j)=\sum_{j=1}^{n}\left\{r_{1, j}-\theta r_{2, j}\right\} y_{2}(j)+\sum_{j=1}^{n}\left\{y_{1}^{\eta}(j)-\theta y_{2}^{\eta}(j)\right\} y_{2}(j)
$$

Applying Theorem 3.1, Assumption 2.6 and the Continuous Mapping Theorem, we obtain

$$
\begin{aligned}
n^{-3 / 2-H} & \sum_{j=1}^{n}\left\{y_{1}^{\eta}(j)-\theta y_{2}^{\eta}(j)\right\} y_{2}(j) \\
& \rightarrow \int_{0}^{1}\left\{\theta^{-1} \sqrt{\lambda_{1}} \sigma_{1, e} B_{1}(t)+\sqrt{\lambda_{2}} \sigma_{2, e} B_{2}(t)\right\}\left\{c_{1} B_{1, H}\left(\lambda_{1} t\right)-\theta c_{2} B_{2, H}\left(\lambda_{2} t\right)\right\} \mathrm{d} t \\
& \stackrel{\operatorname{law}}{=} \Sigma \int_{0}^{1} B(t) B_{H}(t) \mathrm{d} t
\end{aligned}
$$

where $B$ is a standard Brownian motion, $B_{H}$ is a fractional Brownian motion, independent of $B$ and

$$
\Sigma^{2}=\left(\theta^{-2} \lambda_{1} \sigma_{1, e}^{2}+\lambda_{2} \sigma_{2, e}^{2}\right)\left(c_{1}^{2} \lambda_{1}^{2 H}+\theta^{2} c_{2}^{2} \lambda_{2}^{2 H}\right) .
$$

There only remains to prove that, for $i=1,2$,

$$
n^{-3 / 2} \sum_{j=1}^{n} r_{i, j} y_{2}(j)=O_{P}(1)
$$

The convergence of $n^{-1 / 2} y_{2}$ is uniform on $[0,1]$, so $n^{-1 / 2} \max _{1 \leq j \leq n}\left|y_{2}(j)\right|=O_{P}(1)$. Therefore, it suffices to prove that

$$
n^{-1} \sum_{j=1}^{n}\left|r_{i, j}\right|=O_{P}(1)
$$

Recall that $N_{i}(s)<k \Leftrightarrow t_{i, k}>s$. Thus, for $k \leq N_{1}(n)$,

$$
N_{1}\left(t_{2, N_{2}(j)}\right)<k \leq N_{1}(j) \Leftrightarrow t_{2, N_{2}(j)}<t_{1, k} \leq j
$$


The first inequality on the righthand side means that there is no point of $N_{2}$ between $t_{1, k}$ and $j$, i.e. $j \leq t_{2, N_{2}\left(t_{1, k}\right)+1}$. Let $A_{2}(t)=t_{2, N_{2}(t)+1}-t$ denote the forward recurrence time of $N_{2}$, i.e. the time between $t$ and the next event of $N_{2}$ after $t$. Thus,

$$
\sum_{j=1}^{n}\left|r_{1, j}\right| \leq \sum_{j=1}^{n} \sum_{k=N_{1}\left(t_{\left.2, N_{2}(j)\right)+1}\right.}^{N_{1}(j)}\left|e_{1, k}\right|=\sum_{k=1}^{N_{1}(n)}\left|e_{1, k}\right|\left\{A_{2}\left(t_{1, k}\right)+1\right\} .
$$

We thus get the bound for the conditional expectation given the sigma-field $\mathcal{N}$ generated by the counting processes $N_{1}$ and $N_{2}$ :

$$
\mathbb{E}\left[\sum_{j=1}^{n}\left|r_{1, j}\right| \mid \mathcal{N}\right] \leq C \sum_{k=1}^{N_{1}(n)} A_{2}\left(t_{1, k}\right) .
$$

Conditioning on $N_{1}$ and applying (3.3) yields

$$
\mathbb{E}\left[\sum_{j=1}^{n}\left|r_{1, j}\right| \mid N_{1}\right] \leq C N_{1}(n)=O_{P}(n) .
$$

This proves (4.6) and concludes the proof of Theorem 3.2.

Proof of Theorem 3.3. We note first that

$$
\begin{aligned}
n \hat{\sigma}_{\hat{\theta}_{n}^{O L S}}^{2} & =\frac{\sum_{j=1}^{n}\left[y_{1}(j)-\hat{\theta}_{n}^{O L S} y_{2}(j)\right]^{2}}{\sum_{j=1}^{n} y_{2}^{2}(j)}=\frac{\sum_{j=1}^{n}\left[y_{1}(j)-\theta y_{2}(j)+\left(\theta-\hat{\theta}_{n}^{O L S}\right) y_{2}(j)\right]^{2}}{\sum_{j=1}^{n} y_{2}^{2}(j)} \\
& =\frac{\sum_{j=1}^{n}\left[y_{1}(j)-\theta y_{2}(j)\right]^{2}}{\sum_{j=1}^{n} y_{2}^{2}(j)}-\left(\theta-\hat{\theta}_{n}^{O L S}\right)^{2} .
\end{aligned}
$$

Thus,

$$
\frac{n}{t_{n}^{2}}=\frac{1}{n^{1-2 H}\left(\hat{\theta}_{n}^{O L S}-\theta\right)^{2}} \frac{n^{-2 H-1} \sum_{j=1}^{n}\left[y_{1}(j)-\theta y_{2}(j)\right]^{2}}{n^{-2} \sum_{j=1}^{n} y_{2}^{2}(j)}-1 .
$$

Note that

$$
\begin{aligned}
n^{-2 H-1} \sum_{j=1}^{n}\left[y_{1}(j)-\theta y_{2}(j)\right]^{2} \\
=n^{-2 H-1} \sum_{j=1}^{n}\left[y_{1}^{\eta}(j)-\theta y_{2}^{\eta}(j)\right]^{2} \\
\quad+n^{-2 H-1} \sum_{j=1}^{n}\left(r_{1, j}-\theta r_{2, j}\right)^{2}+2 n^{-2 H-1} \sum_{j=1}^{n}\left(r_{1, j}-\theta r_{2, j}\right)\left(y_{1}^{\eta}(j)-\theta y_{2}^{\eta}(j)\right) .
\end{aligned}
$$


By Theorem 3.1 and the Continuous Mapping Theorem, the first term is $O_{P}(1)$. We will prove below that $n^{-1} \sum_{j=1}^{n}\left(r_{1, j}-\theta r_{2, j}\right)^{2}=O_{P}(1)$, implying that the second and last terms are $o_{P}(1)$. By the proof of Theorem 3.2, we also have that

$$
n^{1 / 2-H}\left(\hat{\theta}_{n}^{O L S}-\theta\right)=\frac{n^{-3 / 2-H} \sum_{j=1}^{n}\left(y_{1}^{\eta}(j)-\theta y_{2}^{\eta}(j)\right) y_{2}(j)+o_{P}(1)}{n^{-2} \sum_{j=1}^{n} y_{2}^{2}(j)} .
$$

Thus, we can write

$$
\frac{n}{t_{n}^{2}}=\frac{\left\{n^{-2} \sum_{j=1}^{n} y_{2}^{2}(j)\right\}\left\{n^{-2 H-1} \sum_{j=1}^{n}\left[y_{1}^{\eta}(j)-\theta y_{2}^{\eta}(j)\right]^{2}+o_{P}(1)\right\}}{\left\{n^{-3 / 2-H} \sum_{j=1}^{n}\left[y_{1}^{\eta}(j)-\theta y_{2}^{\eta}(j)\right] y_{2}(j)+o_{P}(1)\right\}^{2}}-1 .
$$

By Theorem 3.1, we know that $\left(n^{-H-1} \sum_{j=1}^{[n \cdot]}\left[y_{1}^{\eta}(j)-\theta y_{2}^{\eta}(j)\right], n^{-1 / 2} y_{2}([n \cdot])\right)$ converge jointly to $\left(\varsigma B, \varsigma_{H} B_{H}\right)$, where $B$ is a standard Brownian motion, $B_{H}$ is a standard fractional motion, mutually independent, and $\varsigma$ and $\varsigma_{H}$ are positive constants. Thus, by the Continuous Mapping Theorem, we have

$$
\frac{n}{t_{n}^{2}} \rightarrow \frac{\int_{0}^{1} B^{2}(t) d t \int_{0}^{1} B_{H}^{2}(t) d t}{\left\{\int_{0}^{1} B(t) B_{H}(t) d t\right\}^{2}}-1
$$

We now deal with the remainder term $n^{-1} \sum_{j=1}^{n}\left(r_{1, j}-\theta r_{2, j}\right)^{2}$. We only prove that $n^{-1} \sum_{j=1}^{n} r_{1, j}^{2}=$ $O_{P}(1)$, the proof for the term involving $r_{2, j}$ being similar. Since the counting processes and the efficient shocks are independent, taking conditional expectations, we obtain

$$
\mathbb{E}\left[\sum_{j=1}^{n} r_{1, j}^{2} \mid \mathcal{N}\right]=\sum_{j=1}^{n} \sum_{k=N_{1}\left(t_{2, N_{2}(j)}\right)+1}^{N_{1}(j)} \sigma_{1, e}^{2}=\sigma_{1, e}^{2} \sum_{k=1}^{N(n)}\left\{A_{2}\left(t_{1, k}\right)+1\right\} .
$$

Sicne the counting processes are mutually independent, we can apply Condition (3.3) to see that the expectation of the sum in the righthand side is $O(n)$. Thus $\sum_{j=1}^{n} r_{1, j}^{2}=O_{P}(n)$ and this concludes the proof.

Proof of Theorem 3.4. We will prove below that there exists a positive constant $C$ such that

$$
m^{2-2 H} s_{m}^{2} \stackrel{\mathbb{P}}{\rightarrow} C
$$

Thus,

$$
\log \left(m^{2-2 H} s_{m}^{2}\right) \stackrel{\mathbb{P}}{\rightarrow} \log C,
$$

and

$$
\hat{H}=1+\frac{\log \left(s_{m}^{2}\right)}{2 \log m}=H+\frac{\log \left(m^{2-2 H} s_{m}^{2}\right)}{2 \log m} \stackrel{\mathbb{P}}{\rightarrow} H .
$$


Proof of (4.7). Elementary algebra yields

$$
m X_{k}^{(m)}=A_{k, m}+V_{k, m}-R_{k, m}
$$

where

$$
\begin{aligned}
A_{k, m}=\sigma_{1}\left\{B_{1, H}\left(N_{1}(k m)\right)\right. & \left.-B_{1, H}\left(N_{1}((k-1) m)\right)\right\} \\
& -\theta \sigma_{2}\left\{B_{2, H}\left(N_{2}(k m)\right)-B_{2, H}\left(N_{2}((k-1) m)\right)\right\} \\
R_{k, m}=\left(\hat{\theta}_{n}^{O L S}-\theta\right)\left[y_{2}(k m)-\right. & \left.y_{2}((k-1) m)\right]
\end{aligned}
$$

The convergence (4.7) is a consequence of the following three convergences.

$$
\begin{aligned}
& m^{1-2 H} n^{-1} \sum_{k=1}^{\lfloor n / m\rfloor} R_{k, m}^{2} \stackrel{\mathbb{P}}{\rightarrow} 0, \\
& m^{1-2 H} n^{-1} \sum_{k=1}^{\lfloor n / m\rfloor} V_{k, m}^{2} \stackrel{\mathbb{P}}{\rightarrow} 0, \\
& m^{1-2 H} n^{-1} \sum_{k=1}^{\lfloor n / m\rfloor} A_{k, m}^{2} \stackrel{\mathbb{P}}{\rightarrow} C .
\end{aligned}
$$

We will only prove (4.10), the other convergences being similarly and more easily obtained. Let $T_{n}=m^{1-2 H} n^{-1} \sum_{k=1}^{\lfloor n / m\rfloor} A_{k, m}^{2}$ and let $\mathcal{N}$ denote the sigma-field generated by the point processes $N_{1}$ and $N_{2}$. We will prove that there exists a positive constant $C$ such that

$$
\begin{gathered}
\lim _{n \rightarrow \infty} \mathbb{E}\left[T_{n} \mid \mathcal{N}\right]=C, \\
\lim _{n \rightarrow \infty} \operatorname{var}\left(T_{n} \mid \mathcal{N}\right)=0 .
\end{gathered}
$$

By the Bienaymé-Chebyshev inequality, (4.11) and (4.12), we have

$$
\begin{aligned}
\mathbb{P}\left(\left|T_{n}-C\right|>\epsilon \mid \mathcal{N}\right) & \leq \epsilon^{-2} \mathbb{E}\left[\left|T_{n}-C\right|^{2} \mid \mathcal{N}\right] \\
& \leq \epsilon^{-2} \operatorname{var}\left(T_{n} \mid \mathcal{N}\right)+\epsilon^{-2}\left(\mathbb{E}\left[T_{n} \mid \mathcal{N}\right]-C\right)^{2} \stackrel{\mathbb{P}}{\rightarrow} 0
\end{aligned}
$$

This precisely means that $T_{n}$ converges to $C$ in conditional probability, i.e. for all $\epsilon>0$,

$$
\lim _{n \rightarrow \infty} \mathbb{P}\left(\left|T_{n}-C\right|>\epsilon \mid \mathcal{N}\right)=0
$$


Since a probability is bounded by one and $\mathbb{P}\left(\left|T_{n}-C\right|>\epsilon\right)=\mathbb{E}\left[\mathbb{P}\left(\left|T_{n}-C\right|>\epsilon \mid \mathcal{N}\right)\right]$, the bounded convergence theorem implies that for all $\epsilon>0$,

$$
\lim _{n \rightarrow \infty} \mathbb{P}\left(\left|T_{n}-C\right|>\epsilon\right)=0
$$

i.e. $T_{n} \stackrel{\mathbb{P}}{\rightarrow} C$.

For simplicity of notation, we also assume that $n / m$ is an integer. For any $a<b$, $N_{i}(a, b]$ denotes the number of points of $N_{i}$ in the interval $(a, b]$. Note that $N_{i}(0, t]=N_{i}(t)$ for all $t>0$. Since $B_{1, H}$ and $B_{2, H}$ are independent of $\mathcal{N}$, we have, for any $s<t$,

$$
\operatorname{var}\left(B_{i, H}\left(N_{i}(t)-B_{i, H}\left(N_{i}(s)\right) \mid \mathcal{N}\right)=\left\{N_{i}(s, t]\right\}^{2 H} .\right.
$$

Since moreover $B_{1, H}$ and $B_{2, H}$ are mutually independent, this yields

$$
\begin{aligned}
\mathbb{E} & {\left[T_{n} \mid \mathcal{N}\right] } \\
& =\sigma_{1}^{2} m^{1-2 H} n^{-1} \sum_{k=1}^{n / m}\left\{N_{1}(((k-1) m, k m])\right\}^{2 H}+\sigma_{2}^{2} \theta^{2} m^{1-2 H} n^{-1} \sum_{k=1}^{n / m}\left\{N_{2}(((k-1) m, k m])\right\}^{2 H} \\
& =\sigma_{1}^{2} T_{1, n}+\sigma_{2}^{2} \theta^{2} T_{2, n} .
\end{aligned}
$$

We will prove that $T_{i, n}$ converges in probability to $\lambda_{i}^{2 H}, i=1,2$, where $\lambda_{i}$ is the intensity of $N_{i}$, i.e. $\mathbb{E}\left[N_{i}(0,1]\right]=\lambda_{i}$. This will imply (4.11) with $C=\sigma_{2}^{2} \lambda_{1}^{2 H}+\sigma_{2}^{2} \theta^{2} \lambda_{2}^{2 H}$.

Since $N_{i}$ is stationary, we have

$$
\mathbb{E}\left[\left|T_{i, n}-\lambda_{i}^{2 H}\right|\right] \leq \mathbb{E}\left[\left|\left\{m^{-1} N_{i}(m)\right\}^{2 H}-\lambda_{i}^{2 H}\right|\right] .
$$

For brevity, we now omit the subscript $i$. By stationarity and ergodicity, $m^{-1} N(m)$ converges almost surely to $\lambda$ as $m$ goes to infinity. Since $0<2 H<1$, it holds that $\left|a^{2 H}-b^{2 H}\right| \leq|a-b|^{2 H}$ for all real numbers $a, b$. Thus, for any $\epsilon>0$,

$$
\begin{aligned}
\mathbb{E} & {\left[\left|\left\{m^{-1} N(m)\right\}^{2 H}-\lambda^{2 H}\right|\right] } \\
& \leq \epsilon^{2 H}+\mathbb{E}\left[\left|\left\{m^{-1} N(m)\right\}^{2 H}-\lambda^{2 H}\right| \mathbf{1}_{\left.\left|m^{-1} N(m)-\lambda\right|>\epsilon\right\}}\right] \\
& \leq \epsilon^{2 H}+\mathbb{E}\left[\left\{m^{-1} N(m)\right\}^{2 H} \mathbf{1}_{\left.\left|m^{-1} N(m)-\lambda\right|>\epsilon\right\}}\right]+\lambda^{2 H} \mathbb{P}\left(\left|m^{-1} N(m)-\lambda\right|>\epsilon\right) .
\end{aligned}
$$

By ergodicity, the last term above tends to zero as $m$ tends to infinity. To deal with the middle term, we apply Hölder's inequality and obtain

$$
\begin{aligned}
\mathbb{E}\left[\left\{m^{-1} N(m)\right\}^{2 H}\right. & \left.\mathbf{1}_{\left.\left|m^{-1} N(m)-\lambda\right|>\epsilon\right\}}\right] \\
& \leq \mathbb{E}^{2 H}\left[m^{-1} N(m)\right] \mathbb{P}^{1-2 H}\left(\left|m^{-1} N(m)-\lambda\right|>\epsilon\right) \\
& =\lambda^{2 H} \mathbb{P}^{1-2 H}\left(\left|m^{-1} N(m)-\lambda\right|>\epsilon\right) \rightarrow 0,
\end{aligned}
$$

as $m \rightarrow \infty$, again by ergodicity. Thus we obtain that $\lim _{n \rightarrow{ }_{i} n f t y} \mathbb{E}\left[\left|T_{i, n}-\lambda_{i}^{2 H}\right|\right]=$ and this concludes the proof of (4.11). 
We now prove (4.12). We denote the conditional variance and covariance given $\mathcal{N}$ by $\operatorname{var}_{N}$ and $\operatorname{cov}_{N}$, respectively. Since $A_{k, m}$ is conditionally Gaussian, we have, for all $k, k^{\prime}$,

$$
\left.\operatorname{cov}_{N}\left(A_{k, m}^{2}, A_{k^{\prime}, m}^{2}\right)\right)=2 \operatorname{cov}_{N}^{2}\left(A_{k, m}, A_{k^{\prime}, m}\right) .
$$

Thus, denoting $S_{n}=\sum_{k=1}^{[n / m]} A_{k, m}^{2}$, we have

$$
\operatorname{var}_{N}\left(S_{n}\right)=2 \sum_{k=1}^{n / m} \operatorname{var}_{N}^{2}\left(A_{k, m}\right)+4 \sum_{k=1}^{n / m-1} \sum_{k^{\prime}=k+1}^{n / m} \operatorname{cov}_{N}^{2}\left(A_{k, m}, A_{k^{\prime}, n}\right)=2 \times I+4 \times I I
$$

Applying (4.13) to compute $\operatorname{var}_{N}\left(A_{k, m}\right)$ and taking expectation, we have, by stationarity,

$$
m^{2-4 H} n^{-2} \mathbb{E}[I]=\sigma_{1}^{2} m n^{-1} \mathbb{E}\left[\left(N_{1}(m) / m\right)^{4 H}\right]+\sigma_{2}^{2} \theta^{2} m n^{-1} \mathbb{E}\left[\left(N_{2}(m) / m\right)^{4 H}\right] .
$$

Since $4 H<2$, by Jensen's inequality, $\mathbb{E}\left[N_{i}^{4 H}(m)\right] \leq\left\{\mathbb{E}\left[N_{i}^{2}(m)\right]\right\}^{2 H}$ and by stationarity $\mathbb{E}\left[N_{i}^{2}(m)\right] \leq m^{2} \mathbb{E}\left[N_{i}^{2}(1)\right]$. Thus $m^{2-4 H} n^{-2} \mathbb{E}[I]=O(m / n)$.

Consider now the last term $I I$. For any positive real numbers $s<t<u<v$ and a standard fractional Brownian motion $H$, we have

$$
\operatorname{cov}\left(B_{H}(t)-B_{H}(s), B_{H}(v)-B_{H}(u)\right)=|v-s|^{2 H}-|v-t|^{2 H}+|u-t|^{2 H}-|u-s|^{2 H} .
$$

Thus,

$$
\begin{aligned}
\operatorname{cov}_{N}\left(A_{k, m}, A_{k^{\prime}, n}\right) & =\sigma_{1}^{2}\left\{N_{1}^{2 H}\left((k-1) m, k^{\prime} m\right]-N_{1}^{2 H}\left(k m, k^{\prime} m\right]\right. \\
& \left.+N_{1}^{2 H}\left(k m,\left(k^{\prime}-1\right) m\right]-N_{1}^{2 H}\left((k-1) m,\left(k^{\prime}-1\right) m\right]\right\} \\
& +\sigma_{2}^{2} \theta^{2}\left\{N_{2}^{2 H}\left((k-1) m, k^{\prime} m\right]-N_{2}^{2 H}\left(k m, k^{\prime} m\right]\right. \\
& \left.+N_{2}^{2 H}\left(k m,\left(k^{\prime}-1\right) m\right]-N_{2}^{2 H}\left((k-1) m,\left(k^{\prime}-1\right) m\right]\right\} \\
& =\sigma_{1}^{2} C_{k, k^{\prime}}+\sigma_{2}^{2} \theta^{2} C_{k, k^{\prime}}^{\prime} .
\end{aligned}
$$

Hereafter, we only deal with the terms related to $N_{1}$, the other terms being similarly dealt with and we omit the subscript 1 . For any $a, c \geq 0$ and $b>0$, since $0<2 H<1$, we have

$$
\begin{aligned}
0 & \leq(a+b)^{2 H}-b^{2 H}-(a+b+c)^{2 H}+(b+c)^{2 H} \\
& =2 H(1-2 H) \int_{b}^{a+b} \int_{z}^{z+c} u^{2 H-2} \mathrm{~d} u \mathrm{~d} z \leq a c b^{2 H-2} .
\end{aligned}
$$

Applying this bound with $a_{k}=N((k-1) m, k m], b_{k, k^{\prime}}=N\left(k m,\left(k^{\prime}-1\right) m\right]$ yields

$$
C_{k, k^{\prime}}^{2} \leq b_{k, k^{\prime}}^{4 H-4} a_{k}^{2} a_{k^{\prime}}^{2} \mathbf{1}_{\left\{b_{k, k^{\prime}}>0\right\}} .
$$

Taking expectation, we have, by stationarity,

$$
\begin{aligned}
m^{2-4 H} n^{-2} & \sum_{1 \leq k<k^{\prime \prime} \leq n / m} \mathbb{E}\left[C_{k, k^{\prime}}^{2} \mathbf{1}_{\left\{b_{k, k^{\prime}}>0\right\}}\right] \\
& \leq m^{1-4 H} n^{-1} \sum_{k=1}^{n / m} \mathbb{E}\left[N^{4 H-4}(k m) \mathbf{1}_{\{N(k m)>0\}} N^{2}(m) N^{2}((k-1) m, k m]\right] .
\end{aligned}
$$


Applying Hölder's inequality yields

$$
\begin{aligned}
m^{2-4 H} n^{-2} & \sum_{1 \leq k<k^{\prime \prime} \leq n / m} \mathbb{E}\left[C_{k, k^{\prime}}^{2} \mathbf{1}_{\left\{b_{k, k^{\prime}}>0\right\}}\right] \\
& \leq m^{-2} \mathbb{E}^{1 / 2}\left[N^{4}(m)\right] \frac{m}{n} \sum_{k=1}^{n / m} \mathbb{E}^{1 / 2}\left[\{N(k m) / k m\}^{8 H-8} \mathbf{1}_{\{N(k m)>0\}}\right] k^{4 H-4} .
\end{aligned}
$$

By stationarity, $\mathbb{E}\left[N^{4}(m)\right]=O\left(m^{4}\right)$, and applying Assumption (3.4), we obtain, for some constant $c$,

$$
m^{2-4 H} n^{-2} \sum_{1 \leq k<k^{\prime \prime} \leq n / m} \mathbb{E}\left[C_{k, k^{\prime}}^{2} 1_{\left\{b_{k, k^{\prime}}>0\right\}}\right] \leq c \frac{m}{n} \sum_{k=1}^{n / m} k^{4 H-4}=o(1) .
$$

Consider now the event $\left\{b_{k, k^{\prime}}=0\right\}$. Then, using the above notations, we have

$$
\mathbb{E}\left[C_{k, k^{\prime}}^{2} \mathbf{1}_{\left\{b_{k, k^{\prime}}=0\right\}}\right]=\mathbb{E}\left[\left\{a_{k}^{2 H}+a_{k^{\prime}}^{2 H}-\left(a_{k}+a_{k^{\prime}}\right)^{2 H}\right\}^{2} \mathbf{1}_{\left\{b_{k, k^{\prime}}=0\right\}}\right] \leq 4 \mathbb{E}\left[\left(a_{k}^{4 H}+a_{k^{\prime}}^{4 H}\right) \mathbf{1}_{\left\{b_{k, k^{\prime}}=0\right\}}\right] .
$$

Thus, by stationarity of $N$, we have

$$
\begin{array}{rl}
m^{2-4 H} n^{-2} \sum_{1 \leq k<k^{\prime \prime} \leq n / m} & \mathbb{E}\left[C_{k, k^{\prime}}^{2} \mathbf{1}_{\left\{b_{k, k^{\prime}}=0\right\}}\right] \\
\leq & m^{1-4 H} n^{-1} \sum_{k=1}^{n / m} \mathbb{E}\left[N^{4 H}(m) \mathbf{1}_{\{N(m, k m]=0\}}\right] \\
& +m^{1-4 H} n^{-1} \sum_{k=1}^{n / m} \mathbb{E}\left[N^{4 H}((k-1) m, k m] \mathbf{1}_{\{N((k-1) m)=0\}}\right] .
\end{array}
$$

Applying Hölder's inequality, $\mathbb{E}\left[N^{2}(m)\right]=O\left(m^{2}\right)$ and stationarity yields

$$
\begin{aligned}
m^{1-4 H} n^{-1} & \sum_{k=1}^{n / m} \mathbb{E}\left[N^{4 H}(m) \mathbf{1}_{\{N(m, k m]=0\}}\right]=m^{1-4 H} n^{-1} \mathbb{E}\left[N^{4 H}(m) \mathbf{1}_{\{N(m, n]=0\}}\right] \\
& \leq m^{1-4 H} n^{-1} \mathbb{E}^{2 H}\left[N^{2}(m)\right] \mathbb{P}^{1-2 H}(N(m, n]=0) \leq C m n^{-1} \mathbb{P}^{1-2 H}(N(n-m)=0) .
\end{aligned}
$$

Since $n / m \rightarrow \infty$, it holds that $\lim _{n \rightarrow \infty} \mathbb{P}(N(n-m)=0)=0$. Similarly,

$$
\begin{aligned}
m^{1-4 H} n^{-1} & \sum_{k=1}^{n / m} \mathbb{E}\left[N^{4 H}((k-1) m, k m] \mathbf{1}_{\{N((k-1) m)=0\}}\right] \\
& \leq m^{1-4 H} n^{-1} \mathbb{E}^{2 H}\left[N^{2}(m)\right] \sum_{k=1}^{n / m} \mathbb{P}^{1-2 H}(N((k-1) m)=0) \leq \mathbb{P}^{1-2 H}(N(n)=0)
\end{aligned}
$$

and this last term tends to 0 as $n$ tends to infinity. This concludes the proof of (4.12) and of (4.7). 
Proof of Theorem 3.5. The proof is a consequence of the convergence (4.2), the decomposition (4.3), and Lemmas 4.2 and 4.3, whose assumptions are those of the Theorem.

Lemma 4.2. Under the assumptions of Theorem 3.5,

$$
n^{-H-1 / 2} \sum_{j=1}^{n}\left\{y_{1}^{\eta}(j)-\theta y_{2}^{\eta}(j)\right\} y_{2}(j) \rightarrow \Sigma_{0} \int_{0}^{1} B(s) \mathrm{d} B_{H}(s) .
$$

where $B_{H}$ is a standard fractional Brownian motion independent of $B$ and

$$
\Sigma_{0}=\left(\theta^{-2} \lambda_{1} \sigma_{1, e}^{2}+\lambda_{2} \sigma_{2, e}^{2}\right)\left(c_{1}^{2}+\theta^{2} c_{2}^{2}\right) .
$$

Proof of Lemma 4.2. Denote $S_{n}=\sum_{j=1}^{n}\left\{y_{1}^{\eta}(j)-\theta y_{2}^{\eta}(j)\right\} y_{2}(j)$ and write $y_{2}=y_{2}^{e}+y_{2}^{\eta}$ with obvious notation. Denote $\zeta_{j}=y_{1}^{\eta}(j)-\theta y_{2}^{\eta}(j)=\xi_{1, N_{1}(j)}-\theta \xi_{2, N_{2}(j)}$. Then

$$
S_{n}=\sum_{j=1}^{n} \zeta_{j} y_{2}^{e}(j)+\sum_{j=1}^{n} \zeta_{j} \xi_{2, N_{2}(j)}
$$

By the last part of Assumption 2.7, the last term in the righthand side of $(4.15)$ is $O_{P}(n)$. Consider the first term in the righthand side of (4.15), say $S_{1, n}$. Write

$$
\begin{aligned}
S_{1, n} & =\sum_{j=1}^{n} \zeta_{j} \sum_{k=1}^{N_{2}(j)} e_{2, k}+\theta^{-1} \sum_{j=1}^{n} \zeta_{j} \sum_{k=1}^{N_{1}\left(t_{2, N_{2}(j)}\right)} e_{1, k} \\
& =\sum_{k=1}^{N_{2}(n)} e_{2, k} \sum_{\left\{j \leq n: N_{2}(j) \geq k\right\}} \zeta_{j}+\theta^{-1} \sum_{k=1}^{N_{1}\left(t_{2, N_{2}(n)}\right)} e_{1, k} \sum_{\left\{j \leq n: N_{1}\left(t_{2, N_{2}(j)}\right) \geq k\right\}} \zeta_{j} \\
& =T_{1, n}+\theta^{-1} T_{2, n} .
\end{aligned}
$$

Denote $W_{n}(t)=\sum_{j=1}^{[n t]} \zeta_{j}$. Since $N_{2}(j)<k$ iff $j<t_{2, k}$, we obtain

$$
T_{1, n}=y_{2}^{e_{2}}(n) W_{n}(1)-\sum_{k=1}^{N_{2}(n)} e_{2, k} W_{n}\left(t_{2, k} / n\right) .
$$

By Assumption 2.7 and Theorem 3.1, $n^{-1 / 2-H} y_{2}^{e_{2}}(n) W_{n}(1) \rightarrow \sqrt{\lambda_{2}} \sigma_{2} B_{2}(1) Z(1)$ with $Z=$ $c_{1} B_{H}^{(1)}-\theta c_{2} B_{H}^{(2)} \stackrel{\text { law }}{=} \sqrt{c_{1}^{2}+\theta^{2} c_{2}^{2}} B_{H}$. Let the last term be denoted by $U_{n}$. Since the shocks $e_{i, k}$ are i.i.d. Gaussian, we can compute the characteristic function of $U_{n}$.

$$
\begin{aligned}
\mathbb{E}\left[\exp \left\{\mathrm{itn} n^{-1 / 2-H} U_{n}\right\}\right] & =\mathbb{E}\left[\exp \left\{-\frac{\sigma_{2, e}^{2} t^{2}}{2} \frac{1}{n} \sum_{k=1}^{N_{2}(n)}\left(n^{-H} W_{n}\left(t_{2, k} / n\right)\right)^{2}\right\}\right] \\
& \rightarrow \mathbb{E}\left[\exp \left\{-\frac{\lambda_{2} \sigma_{2, e}^{2} t^{2}}{2} \int_{0}^{1} Z^{2}(s) \mathrm{d} s\right\}\right] .
\end{aligned}
$$


The convergence is actually joint with that of $n^{-1 / 2-H} y_{2}^{e_{2}}(n) W_{n}$, thus we have

$$
n^{-1 / 2-H} T_{1, n} \rightarrow \sqrt{\lambda_{2}} \sigma_{2, e} B_{2}(1) Z(1)-\sqrt{\lambda_{2}} \sigma_{2, e} \int_{0}^{1} Z(s) \mathrm{d} B_{2}(s) .
$$

The limit can also be written as $\sqrt{\lambda_{2}} \sigma_{2, e} \int_{0}^{1} B_{2}(s) \mathrm{d} Z(s)$. Consider now the term $T_{2, n}$. Note that $N_{1}\left(t_{2, N_{2}(j)}\right)<k$ iff $j \leq t_{2, N_{2}\left(t_{1, k}\right)+1}$. Thus

$$
T_{2, n}=\sum_{k=1}^{N_{1}\left(t_{2, N_{2}(n)}\right)} e_{1, k} W_{n}(1)-\sum_{k=1}^{N_{1}\left(t_{2, N_{2}(n)}\right)} e_{1, k} W_{n}\left(t_{2, N_{2}\left(t_{1, k}\right)+1} / n\right) .
$$

By similar arguments as previously, we obtain

$$
n^{-H-1 / 2} T_{2, n} \rightarrow \sqrt{\lambda_{1}} \sigma_{1} B_{1}(1) Z(1)-\sqrt{\lambda_{1}} \sigma_{1} \int_{0}^{1} Z(s) \mathrm{d} B_{1}(s) .
$$

All convergences hold jointly, thus (4.14) holds.

Lemma 4.3. Under the assumptions of Theorem 3.5,

$$
\sum_{j=1}^{n}\left\{r_{1, j}-\theta r_{2, j}\right\} y_{2}(j)=O_{P}(n)
$$

Proof of Lemma 4.3. We first study the term with $r_{1, j}$ and split it into three parts.

$$
\sum_{j=1}^{n} r_{1, j} y_{2}(j)=\sum_{j=1}^{n} r_{1, j} y_{2}^{e_{1}}(j)+\sum_{j=1}^{n} r_{1, j} y_{2}^{e_{2}}(j)+\sum_{j=1}^{n} r_{1, j} y_{2}^{\eta}(j)
$$

We start with the last one. Recall that $N_{1}\left(t_{2, N_{2}(j)}\right)<k \leq N_{1}(j)$ iff $t_{1, k} \leq j \leq t_{1, k}+A_{2}\left(t_{1, k}\right)$. Thus

$$
\sum_{j=1}^{n} r_{1, j} y_{2}^{\eta}(j)=\sum_{j=1}^{n} \xi_{2, N_{2}(j)} \sum_{N_{1}\left(t_{2, N_{2}(j)}\right)<k \leq N_{1}(j)} e_{1, k}
$$

If the microstructure shocks are independent of the counting processes, then

$$
\begin{aligned}
\mathbb{E} & {\left[\left(\sum_{j=1}^{n} e_{1, k} \sum_{t_{1, k} \leq j<t_{1, k}+A_{2}\left(t_{1}, k\right)} \xi_{2, N_{2}(j)}\right)^{2} \mid \mathcal{N}\right] } \\
& =\sigma_{1, e}^{2} \sum_{k=1}^{N_{1}(n)} \mathbb{E}\left[\left(\sum_{t_{1, k} \leq j<t_{1, k}+A_{2}\left(t_{1}, k\right)} \xi_{2, N_{2}(j)}\right)^{2} \mid \mathcal{N}\right] \leq C \sum_{k=1}^{N_{1}(n)}\left(A_{2}\left(t_{1, k}\right)+1\right)^{2} \sup _{\ell} \mathbb{E}\left[\xi_{2, \ell}^{2}\right] .
\end{aligned}
$$


Conditioning on $N_{1}$ and then taking expectation yields

$\mathbb{E}\left[\left(\sum_{j=1}^{n} e_{1, k} \sum_{t_{1, k} \leq j<t_{1, k}+A_{2}\left(t_{1}, k\right)} \xi_{2, N_{2}(j)}\right)^{2}\right] \leq C \mathbb{E}\left[N_{1}(n)\right] \sup _{t} \mathbb{E}\left[\left\{1+A_{2}(t)\right\}^{2}\right] \sup _{\ell} \mathbb{E}\left[\xi_{\ell}^{2}\right]=O(n)$.

Consider now $R_{2, n}=\sum_{j=1}^{n} r_{1, j} y_{2}^{e_{2}}(j)$.

$$
R_{2, n}=\sum_{j=1}^{n} y_{2}^{e_{2}}(j) \sum_{N_{1}\left(t_{2, N_{2}(j)}\right)+1}^{N_{1}(j)} e_{1, k}=\sum_{k=1}^{N_{1}(n)} e_{1, k} \sum_{t_{1, k} \leq j<t_{1, k}+A_{2}\left(t_{1, k}\right)} y_{2}^{e_{2}}(j) .
$$

By independence of the efficient shocks and the counting processes, we have

$$
\mathbb{E}\left[R_{2, n}^{2} \mid \mathcal{N}\right] \leq C N_{1}(n) \sum_{k=1}^{N_{1}(n)}\left(A_{2}\left(t_{1, k}\right)+1\right)^{2}=O_{P}\left(n^{2}\right) .
$$

This proves that $R_{2, n}=O_{P}(n)$. Consider finally $R_{1, n}=\sum_{j=1}^{n} r_{1, j} y_{2}^{e_{1}}(j)$. By definition, $e_{1, k}$ is independent of $y_{2}^{e_{1}}(j)$ for $j$ such that $N_{1}\left(t_{2, N_{2}(j)}\right)<k$. Thus, we can compute the conditional variance given $\mathcal{N}$.

$$
\begin{aligned}
\mathbb{E}\left[R_{1, n}^{2} \mid \mathcal{N}\right] & =\sigma_{1, e}^{2} \sum_{k=1}^{N_{1}(n)} \mathbb{E}\left[\left(\sum_{t_{1, k} \leq j<t_{1, k}+A_{2}\left(t_{1, k}\right)} y_{2}^{e_{1}}(j)\right)^{2} \mid \mathcal{N}\right] \\
& \leq C N_{2}(n) \sum_{k=1}^{N_{1}(n)}\left(A_{2}\left(t_{1, k}\right)+1\right)^{2}=O_{P}(n)
\end{aligned}
$$

by (3.5). This concludes the proof of Lemma 4.3.

\subsection{Proof of Theorems 3.6 and 3.7}

Write

$$
\tilde{\theta}_{n}=\theta+\frac{\sum_{\ell=1}^{m} d_{\Delta r, \ell} \bar{d}_{\Delta y_{2}, \ell}}{\sum_{\ell=1}^{m}\left|d_{\Delta y_{2}, \ell}\right|^{2}}+\frac{\sum_{\ell=1}^{m} d_{\Delta y^{\eta}, \ell} \bar{d}_{\Delta y_{2}, \ell}}{\sum_{\ell=1}^{m}\left|d_{\Delta y_{2}, \ell}\right|^{2}}
$$

with $y^{\eta}(j)=y_{1}^{\eta}(j)-\theta y_{2}^{\eta}(j), r(j)=r_{1}(j)-\theta r_{2}(j)$ and

$$
r_{1}(j)=\sum_{k=N_{1}\left(t_{2, N_{2}(j)}\right)+1}^{N_{1}(j)} e_{1, k}, \quad r_{2}(j)=\sum_{k=N_{2}\left(t_{1, N_{1}(j)}\right)+1}^{N_{2}(j)} e_{2, k} .
$$

By summation by parts, since $h(0)=h(1)=0$, for any time series $\left\{x_{j}\right\}$, we can write

$$
d_{\Delta x, \ell}=\sum_{j=0}^{n-1}\left\{h_{\ell}(j / n)-h_{\ell}((j+1) / n)\right\} x_{j}=-\frac{1}{n} \sum_{j=0}^{n-1} w_{\ell}(j, n) x_{j}
$$


with $w_{\ell}(j, n)=n\left\{h_{\ell}((j+1) / n)-h_{\ell}(j / n)\right\}$. Applying (4.18) to $y_{2}$ yields

$$
d_{\Delta y_{2}, \ell}=-\frac{1}{n} \sum_{j=0}^{n-1} w_{\ell}(j, n) y_{2}(j)
$$

Since the assumptions of Theorems 3.6 and 3.7 imply those of Theorem 3.1, the Continuous Mapping Theorem yields

$$
\left\{n^{-1 / 2} d_{\Delta y_{2}, \ell}, 1 \leq \ell \leq m\right\} \rightarrow\left\{-\Sigma_{e} \int_{0}^{1} h_{\ell}^{\prime}(s) B(s) \mathrm{d} s, 1 \leq \ell \leq m\right\}
$$

where $B$ is a standard Brownian motion and $\Sigma_{e}^{2}=\theta^{-2} \lambda_{1} \sigma_{1, e}^{2}+\lambda_{2} \sigma_{2, e}^{2}$. By integration by parts, the integral can also be expressed as

$$
-\int_{0}^{1} h_{\ell}^{\prime}(s) B(s) \mathrm{d} s=\int_{0}^{1} h_{\ell}(s) \mathrm{d} B(s) .
$$

This in turn implies

$$
n^{-1} \sum_{\ell=1}^{m}\left|d_{\Delta y_{2}, \ell}\right|^{2} \rightarrow \Sigma_{e}^{2} \sum_{\ell=1}^{m}\left|\int_{0}^{1} h_{\ell}(s) \mathrm{d} B(s)\right|^{2} .
$$

Applying now (4.18) to $y^{\eta}$ we obtain

$$
d_{\Delta y^{\eta}, \ell}=-\frac{1}{n} \sum_{j=0}^{n-1} w_{\ell}(j, n)\left\{y_{1}^{\eta}(j)-\theta y_{2}^{\eta}(j)\right\}
$$

In the case of weak fractional cointegration, we apply Assumption 2.6, the Continuous Mapping Theorem and integration by parts to obtain

$$
n^{-H} \ell(n) d_{\Delta y^{\eta}, \ell}=-n^{-1-H} \ell(n) \sum_{j=0}^{n-1} w_{\ell}(j, n)\left\{y_{1}^{\eta}(j)-\theta y_{2}^{\eta}(j)\right\} \rightarrow \int_{0}^{1} h_{\ell}(t) \mathrm{d} Z_{H}(t)
$$

where, by independence of $B_{H}^{(1)}$ and $B_{H}^{(2)}$,

$$
Z_{H}(t)=c_{1} B_{H}^{(1)}\left(\lambda_{1} t\right)-\theta c_{2} B_{H}^{(2)}\left(\lambda_{2} t\right) \stackrel{\operatorname{law}}{=} \sqrt{\lambda_{1}^{2 H} c_{1}^{2}+\lambda_{2}^{2 H} \theta^{2} c_{2}^{2}} B_{H}
$$

and $B_{H}$ is a standard fractional Brownian motion. The first part of Lemma 4.4 shows that $d_{\Delta r, \ell}$ is negligible under the assumptions of Theorem 3.6. This, and the convergences (4.19), (4.20) and (4.21) conclude the proof of Theorem 3.6.

We now prove Theorem 3.7. Since $h_{\ell}(0)=h_{\ell}(1)=0$, we have $\sum_{j=0}^{n-1} w_{\ell}(j, n)=0$, hence

$$
\sum_{j=0}^{n-1} w_{\ell}(j, n) y_{i}^{\eta}(j)=\sum_{j=0}^{n-1} w_{\ell}(j, n)\left(\xi_{i, N_{i}(j)}-\xi_{i, 0}\right)=\sum_{j=0}^{n-1} w_{\ell}(j, n)\left(\xi_{i, N_{i}(j)}-\mu_{i}^{*}\right) .
$$


Denote $S_{i, 0}=0$ and for $k \geq 1, S_{i, k}=\sum_{j=1}^{k}\left(\xi_{i, N_{i}(j)}-\mu_{i}^{*}\right)$. Define $\omega_{\ell}(j, n)=n\left\{w_{\ell}(j+1, n)-\right.$ $\left.w_{\ell}(j, n)\right\}$. Applying again summation by parts, we have

$$
\sum_{j=0}^{n-1} w_{\ell}(j, n) y_{i}^{\eta}(j)=-\frac{1}{n} \sum_{j=1}^{n-1} \omega_{\ell}(j, n) S_{i, j}+w_{\ell}(n, n) S_{i, n-1}+w_{\ell}(0, n)\left(\xi_{i, 0}-\mu_{i}^{*}\right),
$$

Under Assumption 2.7, by the Continuous Mapping Theorem, we obtain

$$
\begin{aligned}
n^{1-\gamma} \ell(n) d_{\Delta y^{\eta}, \ell} & =-n^{-\gamma} \ell(n) \sum_{j=1}^{n} w_{\ell}(j, n) y_{i}^{\eta}(j) \\
& \rightarrow \int_{0}^{1} h_{\ell}^{\prime \prime}(t) B_{H}^{(i)}(t) \mathrm{d} t-h^{\prime}(1) B_{H}^{(i)}(1) \stackrel{\text { law }}{=}-\int_{0}^{1} h_{\ell}^{\prime}(s) \mathrm{d} B_{H}^{(i)}(s) .
\end{aligned}
$$

The second part of Lemma 4.4 implies that the term $d_{\Delta r, \ell}$ does not contribute to the limit under the Assumptions of Theorem 3.7. This, and the convergences (4.19), (4.20) and (4.22) conclude the proof of Theorem 3.7.

Lemma 4.4. Under the assumptions of Theorem 3.6, then $d_{\Delta r, \ell}=O_{P}(1)$. Under the assumptions of Theorem 3.7, then $d_{\Delta r, \ell}=O_{P}\left(n^{-1 / 2}\right)$.

Proof. Applying (4.18) to $r$, we see that we only need to prove that the independence between the counting processes and the efficient shocks and (3.3) implies that $\sum_{j=1}^{n} w_{\ell}(j, n) r_{i, j}=$ $O_{p}(n)$ and (3.5) implies that $\sum_{j=1}^{n} w_{\ell}(j, n) r_{i, j}=O_{p}\left(n^{1 / 2}\right)$. We start with $r_{1}$.

$$
\sum_{j=1}^{n} w_{\ell}(j, n) r_{1, j}=\sum_{k=1}^{N_{1}(n)} e_{1, k} \sum_{t_{1, k} \leq j<t_{1, k}+A_{2}\left(t_{1, k}\right)} w_{\ell}(j, n) .
$$

Taking conditional expectation yields, for $q=1,2$,

$$
\mathbb{E}\left[\left|\sum_{j=1}^{n} w_{\ell}(j, n) r_{1, j}\right|^{q} \mid \mathcal{N}\right] \leq C \sum_{k=1}^{N_{1}(n)}\left(A_{2}\left(t_{1, k}\right)+1\right)^{q} .
$$

Applying (3.3) if $q=1$ and (3.5) if $q=2$ shows that the last term is $O_{P}(n)$. This proves that $\sum_{j=1}^{n} w_{\ell}(j, n) r_{1, j}=O_{P}(n)$ under the assumptions of Theorem 3.6 and $O_{P}(\sqrt{n})$ under the assumptions of Theorem 3.7. The term $\sum_{j=1}^{n} w_{\ell}(j, n) r_{2, j}$ is dealt with similarly.

\subsection{Proof of Theorem 3.8}

Write

$$
\tilde{\theta}_{n, \delta}=\theta+\frac{\sum_{\ell=1}^{m} d_{\Delta \tilde{r}, \ell} \bar{d}_{\Delta \tilde{y}_{2}, \ell}}{\sum_{\ell=1}^{m}\left|d_{\Delta \tilde{y}_{2}, \ell}\right|^{2}}+\frac{\sum_{\ell=1}^{m} d_{\Delta \tilde{y}^{\eta}, \ell} \bar{d}_{\Delta \tilde{y}_{2}, \ell}}{\sum_{\ell=1}^{m}\left|d_{\Delta \tilde{y}_{2}, \ell}\right|^{2}}
$$


with $\tilde{y}(j)=\tilde{y}_{1}^{\eta}(j)-\theta \tilde{y}_{2}^{\eta}(j), \tilde{r}(j)=\tilde{r}_{1}(j)-\theta \tilde{r}_{2}(j)$ and

$$
r_{1}(s)=\sum_{k=N_{1}\left(t_{2, N_{2}(s)}\right)+1}^{N_{1}(s)} e_{1, k}, \quad r_{2}(s)=\sum_{k=N_{2}\left(t_{1, N_{1}(s)}\right)+1}^{N_{2}(s)} e_{2, k}
$$

and the DFT is defined as in (3.7). Applying summation by parts as in (4.18), we obtain $d_{\Delta \tilde{y}_{2}, \ell}=-\frac{1}{n} \sum_{j=0}^{n-1} w_{\ell}(j, n) \tilde{y}_{2}(j)=-\frac{1}{n} \int_{0}^{n \delta} w_{\ell}(\lceil s / \delta\rceil, n) y_{2}(s) \mathrm{d} s=-\int_{0}^{\delta} w_{\ell}(\lceil n t / \delta\rceil, n) y_{2}(n s) \mathrm{d} t$,

with $w_{\ell}(j, n)=n\left\{h_{\ell}((j+1) / n)-h_{\ell}(j / n)\right\}$ as before, and $\lceil t\rceil$ is the smallest integer larger than or equal to $t$. This yields

$$
n^{-1 / 2} d_{\tilde{y}_{2}, \ell} \rightarrow-\Sigma_{e} \int_{0}^{1} h_{\ell}^{\prime}(s) B(\delta s) \mathrm{d} s \stackrel{\operatorname{law}}{=} \Sigma_{e} \int_{0}^{1} h_{\ell}(s) \mathrm{d} B(s) .
$$

Since $\eta_{j}=\xi_{j}-\xi_{j-1}$, we have

$$
\tilde{y}_{i}^{\eta}(j)=\int_{(j-1) \delta}^{j \delta} \xi_{i, N_{i}(s)} \mathrm{d} s-\delta \xi_{i, 0}
$$

Differencing cancels the term $\delta \xi_{0}$. Applying (4.18) and summation by parts and the property that $\sum_{j=0}^{n-1} w_{\ell}(j, n)=0$, we obtain

$$
\begin{aligned}
d_{\Delta \tilde{y}_{i}^{\eta}, \ell} & =-\frac{1}{n} \sum_{j=0}^{n-1} w_{\ell}(j, n) \int_{(j-1) \delta}^{j \delta} \xi_{i, N_{i}(s)} \mathrm{d} s=-\frac{1}{n} \sum_{j=0}^{n-1} w_{\ell}(j, n) \int_{(j-1) \delta}^{j \delta}\left\{\xi_{i, N_{i}(s)}-\mu_{i}^{*}\right\} \mathrm{d} s \\
& =\frac{1}{n^{2}} \sum_{j=1}^{n-1} \omega_{\ell}(j, n) \int_{0}^{j \delta}\left\{\xi_{i, N_{i}(s)}-\mu_{i}^{*}\right\} \mathrm{d} s-\frac{1}{n} w_{\ell}(n, n) \int_{0}^{(n-1) \delta}\left\{\xi_{i, N_{i}(s)}-\mu_{i}^{*}\right\} \mathrm{d} s .
\end{aligned}
$$

Under Assumption 2.8, we thus have, with $Z=B_{H}^{(1)}-\theta B_{H}^{(2)}$,

$$
n^{1-H}\left\{d_{\Delta \tilde{y}_{1}^{\eta}, \ell}-\theta d_{\Delta \tilde{y}_{2}^{\eta}, \ell}\right\} \rightarrow \int_{0}^{1} h_{\ell}^{\prime \prime}(s) Z(\delta s) \mathrm{d} s-h^{\prime}(1) Z(\delta) .
$$

We must now deal with the remaining terms of the cointegrating error. If $H>1 / 2$, Lemma 4.5 implies that the term $d_{\Delta \tilde{r}, \ell}$ does not contribute to the limit. If $H=1 / 2$, both terms are of the same order. This concludes the proof of Theorem 2.8.

Lemma 4.5. Under the assumptions of Theorem 3.8

$$
d_{\Delta \tilde{r}_{i}, \ell}=O_{P}\left(n^{-1 / 2}\right) .
$$


Proof. Applying as usual summation by parts, we obtain

$$
\begin{aligned}
d_{\Delta \tilde{r}_{1}, \ell} & =-\frac{1}{n} \sum_{k=1}^{N_{1}(n \delta)} e_{1, k} \sum_{j=1}^{n} w_{\ell}(j, n) \int_{(j-1) \delta}^{j \delta} \mathbf{1}_{\left\{t_{1, k} \leq s<t_{1, k}+A_{2}\left(t_{1, k}\right)\right\}} \mathrm{d} s \\
& =-\frac{1}{n} \sum_{k=1}^{N_{1}(n \delta)} e_{1, k} \int_{0}^{n \delta} w_{\ell}(\lceil s / \delta\rceil, n) \mathbf{1}_{\left\{t_{1, k} \leq s<t_{1, k}+A_{2}\left(t_{1, k}\right)\right\}} \mathrm{d} s \\
& =-\frac{1}{n} \sum_{k=1}^{N_{1}(n \delta)} e_{1, k} \int_{t_{1, k} \wedge(n \delta)}^{\left\{t_{1, k}+A_{2}\left(t_{1, k}\right)\right\} \wedge(n \delta)} w_{\ell}(\lceil s / \delta\rceil, n) \mathrm{d} s \\
& =-\sum_{k=1}^{N_{1}(n \delta)} e_{1, k} \int_{\left(t_{1, k} / n\right) \wedge \delta}^{\left\{\left(t_{1, k}+A_{2}\left(t_{1, k}\right)\right) / n\right\} \wedge \delta} w_{\ell}(\lceil n t / \delta\rceil, n) \mathrm{d} t .
\end{aligned}
$$

Taking conditional expectation and applying (3.5), we obtain

$$
\mathbb{E}\left[\left|d_{\Delta \tilde{r}_{1}, \ell}\right|^{2} \mid \mathcal{N}\right] \leq \frac{C}{n^{2}} \sum_{k=1}^{N(n)} A_{2}^{2}\left(t_{1, k}\right)=O_{P}\left(n^{-1}\right) .
$$

\subsection{Additional Lemmas}

Lemma 4.6. If the durations $t_{i, k}-t_{i, k-1}$ form a stationary ergodic sequence with finite moment of order $2 p+1$, if $\mathbb{P}\left(t_{i, 1}>0\right)=1$ and if the associated point process has finite intensity, then

$$
\sup _{s \geq 0} \mathbb{E}\left[\left(t_{i, N_{i}(s)+1}-s\right)^{p}\right]<\infty .
$$

Proof of Lemma 4.6. We omit the index $i$. Let $\theta_{t}$ denote the shift operator and let $A(t)$ be the forward recurrence time. Then $A(s)=t_{N(s)+1}-s=t_{1} \circ \theta_{s}$. Since the sequence $\left\{\tau_{i}\right\}$ is stationary under $\mathbb{P}$, there exists a probability law $P^{*}$ such that $N$ is a stationary ergodic point process under $P^{*}$, see Baccelli and Brémaud (2003, Section 1.3.5). Applying Baccelli and Brémaud (2003, Formula 1.3.3), we obtain

$$
\begin{aligned}
\mathbb{E}\left[A^{p}(s)\right] & =\lambda^{-1} \mathbb{E}^{*}\left[\sum_{k=1}^{N(1)} t_{1}^{p} \circ \theta_{s} \circ \theta_{t_{k}}\right]=\lambda^{-1} \mathbb{E}^{*}\left[\sum_{k=1}^{N(1)} A^{p}\left(s+t_{k}\right)\right] \\
& =\lambda^{-1} \mathbb{E}^{*}\left[\sum_{k=1}^{N(1)}\left\{t_{N\left(s+t_{k}\right)+1}-s-t_{k}\right\}^{p}\right] \leq \lambda^{-1} \mathbb{E}^{*}\left[\sum_{k=1}^{N(1)}\left\{t_{N(s+1)+1}-s\right\}^{p}\right] \\
& =\lambda^{-1} \mathbb{E}^{*}\left[N(1)\left\{t_{N(s+1)+1}-s\right\}^{p}\right] \leq \lambda^{-1}\left\{\mathbb{E}^{*}\left[N(1)^{2}\right]\right\}^{1 / 2}\left\{\mathbb{E}^{*}\left[\left(t_{N(s+1)+1}-s\right)^{2 p}\right]\right\}^{1 / 2} .
\end{aligned}
$$


Since $N$ is stationary under $P^{*}$, the last term does not depend on $s$, and by the RyllNardzewski inversion formula (Baccelli and Brémaud, 2003, Formula 1.2.25), we have

$$
\mathbb{E}^{*}\left[\left(t_{N(s+1)+1}-s\right)^{2 p}\right]=\mathbb{E}^{*}\left[\left(t_{1}+1\right)^{2 p}\right]=\lambda \mathbb{E}\left[\int_{0}^{t_{1}}\left(t_{1}+1-s\right)^{2 p} \mathrm{~d} s \leq \lambda \mathbb{E}\left[\left(1+t_{1}\right)^{2 p+1}\right]\right.
$$

By Baccelli and Brémaud (2003, Property 1.6.3), the point process $N$ is stationary and ergodic under $P^{*}$ since the sequence of durations $\tau_{k}$ is stationary and ergodic. Thus, by Daley and Vere-Jones (2003, Theorem 3.5.III), $\mathbb{E}^{*}\left[N(0,1)^{2}\right]<\infty$. Plugging the last two bounds into (4.24), we obtain that $\mathbb{E}\left[A^{p}(s)\right]$ is uniformly bounded.

Lemma 4.7. Assume that there exists an increasing sequence $\left\{s_{n}, n \geq 0\right\}$ such that $s_{0}=0$ and

(a) $f$ is either constant or strictly increasing and differentiable on $\left(s_{n}, s_{n+1}\right)$ and the jumps of $f$ occur at some (but not necessarily all) of the $s_{n}$;

(b) if $f$ is eiter constant or increasing on both intervals $\left(s_{n}, s_{n+1}\right)$ and $\left(s_{n+1}, s_{n+2}\right)$, then $f$ has a jump at $s_{n+1}$.

Assume moreover that

- (minimum duration of trading and nontrading periods) there exists $\delta_{0}>0$ such that $s_{n+1}-s_{n} \geq \delta_{0}$ for all $n \geq 0$;

- (maximum duration of nontrading periods) there exists $C_{0}$ such that for all $n \geq 0$, if $f$ is constant on $\left(s_{n}, s_{n+1}\right)$, then $s_{n+1}-s_{n} \leq C_{0}$;

- (non stoppage of time during trading periods) there exists $\delta_{1}>0$ such that for all $n \geq 0, f$ is either constant on $\left(s_{n}, s_{n+1}\right)$, or $f^{\prime}(t) \geq \delta_{1}$ for all $t \in\left(s_{n}, s_{n+1}\right)$.

Let $\tilde{N}$ be a point process with event times $\left\{\tilde{t}_{k}\right\}$ and let $N$ be the point process defined by $N(\cdot)=\tilde{N}(f(\cdot))$ with event times $\left\{t_{k}\right\}$. If $\sup _{s \geq 0} \mathbb{E}\left[\left(\tilde{t}_{\tilde{N}(s)+1}-s\right)^{p}\right]<\infty$, then $\sup _{s \geq 0} \mathbb{E}\left[\left(t_{N(s)+1}-s\right)^{p}\right]<\infty$.

Proof of Lemma 4.7. Define the nondecreasing left-continuous inverse $f^{\leftarrow}$ of a nondecreasing càdlàg function $f$ by

$$
f^{\leftarrow}(u)=\inf \{t \mid f(t) \geq u\} .
$$

Note first that $f^{\leftarrow}(u) \leq t$ if only if $u \leq f(t)$ and $f^{\leftarrow}(f(t)) \leq t$. Thus we see that

$$
\begin{aligned}
f^{\leftarrow}\left(\tilde{t}_{n}\right) \leq t & \Leftrightarrow \tilde{t}_{n} \leq f(t) \\
& \Leftrightarrow \tilde{N}(f(t)) \geq n \\
& \Leftrightarrow N(t) \geq n .
\end{aligned}
$$

This characterizes the sequence $\left\{t_{n}\right\}$, thus we obtain that $t_{n}=f^{\leftarrow}\left(\tilde{t}_{n}\right)$. The assumptions on $f$ imply the following properties of $f^{\leftarrow}$. 
- The jumps of $f^{\leftarrow}$ correspond to the intervals $\left(s_{n}, s_{n+1}\right)$ where $f$ is constant. More precisely, if $f$ is constant on $\left(s_{n}, s_{n+1}\right)$, then $f^{\leftarrow}$ has a jump at $f\left(s_{n}\right)$ of size $s_{n+1}-s_{n}$. Since $f^{\leftarrow}$ is left continuous, it holds that

$$
f^{\leftarrow}\left(f\left(s_{n}\right)\right)=s_{n}, \lim _{u \rightarrow f\left(s_{n}\right), u>f\left(s_{n}\right)}=s_{n+1} .
$$

Thus the jumps of $f^{\leftarrow}$ are of size $C_{0}$ at most.

- If $f$ is increasing on an interval $\left(s_{n}, s_{n+1}\right)$, then $f^{\leftarrow}$ is differentiable on $\left(f\left(s_{n}\right), f\left(s_{n}^{-}\right)\right)$ and $\left(f^{\leftarrow}\right)^{\prime}(t) \leq \delta_{1}^{-1}$ for all $t \in\left(f\left(s_{n}\right), f\left(s_{n}^{-}\right)\right)$.

- The jumps of $f$ create no singularity in $f^{\leftarrow}$. If $f\left(s_{n}\right)>f\left(s_{n}^{-}\right)$, then $f^{\leftarrow}$ is constant on the interval $\left(f\left(s_{n}^{-}\right), f\left(s_{n}\right)\right)$.

Let $\lceil x\rceil$ denote the smallest integer greater than or equal to the real number $x$. Then, for $0 \leq s \leq t$

$$
0 \leq f^{\leftarrow}(t)-f^{\leftarrow}(s) \leq C_{0}\left\lceil\frac{t-s}{\delta_{0}}\right\rceil+\delta_{1}^{-1}(t-s) .
$$

Thus, there exits constants $c_{1}, c_{2}$ such that for all $s \leq t$,

$$
0 \leq f(t)-f(s) \leq c_{1}+c_{2}(t-s) .
$$

Consider now the forward recurrence time of the point process $N$. Then

$$
\begin{aligned}
0 & \leq t_{N(s)+1}-s=f^{\leftarrow}\left(\tilde{t}_{\tilde{N}(s)+1}\right)-f^{\leftarrow}(f(s))+f^{\leftarrow}(f(s))-s \\
& \leq f^{\leftarrow}\left(\tilde{t}_{\tilde{N}(f(s))+1}\right)-f^{\leftarrow}(f(s)) \leq c_{1}+c_{2}\left\{\tilde{t}_{\tilde{N}(f(s))+1}-f(s)\right\} .
\end{aligned}
$$

Thus, there exists constants $c_{3}$ and $c_{4}$ such that

$$
\sup _{s \geq 0} \mathbb{E}\left[\left(t_{N(s)+1}-s\right)^{p}\right] \leq c_{3}+c_{4} \sup _{s \geq 0} \mathbb{E}\left[\left(\tilde{t}_{\tilde{N}(s)+1}-s\right)^{p}\right]
$$

Lemma 4.8. Let $\left\{\epsilon_{k}\right\}$ be a sequence of i.i.d. positive random variables with finite mean $\mu_{\epsilon}$. Let $\left\{Y_{k}\right\}$ be a stationary standard Gaussian process such that

$$
\operatorname{cov}\left(Y_{0}, Y_{k}\right)=\ell(n) n^{2 H-2}
$$

for $H \in(1 / 2,1)$ and $\ell$ a slowly varying function. For $k \geq 1$, define

$$
\tau_{k}=\epsilon_{k} \mathrm{e}^{\sigma Y_{k}} .
$$

Then the sequence $\left\{\tau_{k}\right\}$ is ergodic and Assumption 2.1 holds with $\lambda^{-1}=\mu_{\epsilon} \mathrm{e}^{\sigma^{2} / 2}$. If $\mathbb{P}\left(\epsilon_{1}>\right.$ $0)=1$ the Assumption 2.2 holds with $\mu=\lambda=\mu_{\epsilon}^{-1} \mathrm{e}^{-\sigma^{2} / 2}$. If moreover $\mathbb{E}\left[\epsilon_{1}^{q}\right]<\infty$ for all $q \geq 1$, then (3.3) and (3.5) hold. 
Remark 4.1. If instead of (4.25) we assume that

$$
\sum_{k=1}^{\infty}\left|\operatorname{cov}\left(Y_{0}, Y_{k}\right)\right|<\infty
$$

then the moment requirement can be relaxed to $\mathbb{E}\left[\epsilon_{1}^{3}\right]<\infty$ to obtain $(3.3)$ and $\mathbb{E}\left[\epsilon_{1}^{5}\right]<\infty$ to obtain (3.5).

Proof of Lemma 4.8. Note first that $\mathbb{E}\left[\tau_{k}^{p}\right]<\infty$ as long as $\mathbb{E}\left[\epsilon_{1}^{p}\right]<\infty$. By Lemma 4.6, in order to check condition (3.3), we must only prove that the induced point process has finite intensiy, i.e. there exists $t>0$ such that $\mathbb{E}[N(t)]<\infty$. See Baccelli and Brémaud (2003, Section 1.3.5). Note that

$$
\mathbb{E}[N(x)]=\sum_{k=1}^{\infty} \mathbb{P}(N(x) \geq k)=\sum_{k=1}^{\infty} \mathbb{P}\left(t_{k} \leq x\right)
$$

Thus, it suffices to prove that the series on the righthand side is summable. Denote $\mu=\mathbb{E}\left[\tau_{k}\right]$ and $\rho_{n}=\operatorname{cov}\left(Y_{0}, Y_{n}\right)$. Applying Deo et al. (2009b, Proposition 1), we have

$$
\mathbb{E}\left[\left|\sum_{k=1}^{n} \tau_{k}-n \mu\right|^{p}\right]=O\left(v_{n}^{p}\right)
$$

with $v_{n}=n^{H} \ell(n)$. If $\mathbb{E}\left[\epsilon_{1}^{p}\right]<\infty$ for $p$ such that $p(1-H)>1$, for $n$ such that $n \mu>x$, it holds that

$$
\mathbb{P}\left(t_{k} \leq x\right)=O\left(x^{-1} v_{k}^{p}\right)
$$

and this series is summable.

Lemma 4.9. Assume that $\left\{\tau_{k}\right\}$ and $\left\{\xi_{k}\right\}$ are mutually independent stationary sequences such that $\mathbb{E}\left[\xi_{k}\right]=0, \mathbb{E}\left[\tau_{k}^{2}\right]<\infty$ and $\mathbb{E}\left[\xi_{k}^{2}\right]<\infty$. Assume that the sequence of durations is weakly stationary and that $\operatorname{cov}\left(\tau_{0}, \tau_{n}\right)=0\left(n^{-\delta}\right)$ for some $\delta>0$ and $\sup _{s \geq 0} \mathbb{E}\left[t_{N(s)+1}-s\right]<$ $\infty$. Assume that $\operatorname{cov}\left(\xi_{1}, \xi_{n}\right) \sim c n^{2 H-2}$, with $H \in(1 / 2,1)$ and $c>0$, and that

$$
n^{-H} \sum_{k=1}^{[n \cdot]} \xi_{k} \Rightarrow c^{\prime} B_{H}
$$

for some $c^{\prime}>0$. Then

$$
n^{-H} \int_{0}^{T t} \xi_{N(s)} \mathrm{d} s \Rightarrow c^{\prime \prime} B_{H}(t)
$$

for some $c^{\prime \prime}>0$. 
Proof of Lemma 4.9. Denote $\mathbb{E}\left[\tau_{k}\right]=\mu>0$.

$$
\begin{aligned}
\int_{0}^{T} \xi_{N(s)} \mathrm{d} s & =\sum_{k=0}^{N(T)} \tau_{k+1} \xi_{k}-\left(t_{N(T)+1}-T\right) \xi_{N(T)+1} \\
& =\sum_{k=0}^{N(T)}\left(\tau_{k+1}-\mu\right) \xi_{k}+\mu \sum_{k=0}^{N(T)} \xi_{k}-\left(t_{N(T)+1}-T\right) \xi_{N(T)+1}
\end{aligned}
$$

By independence of $\left\{\tau_{k}\right\}$ and $\left\{\xi_{k}\right\}$, we have (assuming without loss of generality that $2 H-\delta>1)$,

$$
\operatorname{var}\left(\sum_{k=0}^{n}\left(\tau_{k+1}-\mu\right) \xi_{k}\right)=O\left(n^{2 H-\delta}\right) .
$$

Thus, $n^{-H} \sum_{k=0}^{[n \cdot]}\left(\tau_{k+1}-\mu\right) \xi_{k} \Rightarrow 0$. Hence by the continuous mapping theorem, it also holds that $n^{-H} \sum_{k=0}^{N(T \cdot)}\left(\tau_{k+1}-\mu\right) \xi_{k} \Rightarrow 0$. By independence and by assumption, $\left(t_{N(t)+1}-T\right) \xi_{N(T)}=$ $O_{P}(1)$. By the continuous mapping theorem, $n^{-H} \sum_{k=0}^{N(T t)} \xi_{k} \Rightarrow c^{\prime} B_{H}\left(\mu^{-1} t\right)$.

Lemma 4.10. Let $\left\{\tau_{k}\right\},\left\{V_{k}\right\}$ and $\left\{\zeta_{k}\right\}$ be sequences of random variables such that

- $\left\{\zeta_{k}\right\}$ is an i.i.d. sequence of zero-mean and unit variance random variables; $\left\{\tau_{k}\right\}$ and $\left\{V_{k}\right\}$ are sequences of positive random variables;

- the sequences $\left\{\left(\tau_{k}, V_{k}\right)\right\}$ and $\left\{\zeta_{k}\right\}$ are mutually independent;

- there exists $s>0$ such that $n^{-1} \sum_{k=1}^{n} \tau_{k+1}^{2} V_{k}^{2} \stackrel{\mathbb{P}}{\rightarrow} s^{2}$;

- $\sup _{k \geq 0} \mathbb{E}\left[\tau_{k+1}^{2+\varepsilon} V_{k}^{2+\varepsilon}\right]<\infty$ for some $\varepsilon>0$;

- $\sup _{s \geq 0} \mathbb{E}\left[t_{N(s)+1}-s\right]<\infty$.

Define $\xi_{k}=\zeta_{k} V_{k}$. Then $T^{-1 / 2} \int_{0}^{T \cdot} \xi_{N(s)} \mathrm{d} s \Rightarrow c B$ for some $c>0$.

Proof. Let $\mathcal{F}_{k}$ be the sigma-field generated by random variables $\left\{\tau_{j+1}, \zeta_{j}, V_{j}, j \leq k\right\}$. Then $\mathbb{E}\left[\xi_{k} \tau_{k+1} \mid \mathcal{F}_{k-1}\right]=\tau_{k+1} V_{k} \mathbb{E}\left[\zeta_{k}\right]=0$. Thus, $\left\{\tau_{k+1} \xi_{k}\right\}$ is a martingale difference sequence. Under the stated assumptions, the martingale invariance principle Hall and Heyde (1980, Theorem 4.1) yields that $n^{-1 / 2} \sum_{k=1}^{[n \cdot]} \tau_{k+1} \xi_{k} \Rightarrow c B$ for some $c>0$. As in the proof of Lemma 4.9, denote $\mathbb{E}\left[\tau_{k}\right]=\mu>0$ and write

$$
\int_{0}^{T} \xi_{N(s)} \mathrm{d} s=\sum_{k=0}^{N(T)} \tau_{k+1} \xi_{k}+\left(t_{N(T)+1}-T\right) \xi_{N(T)} .
$$

By the continuous mapping theorem, we have that $T^{-1 / 2} \sum_{k=1}^{N(T \cdot)} \tau_{k} \xi_{-1} \Rightarrow \lambda c B$. As previously, the last term is a negligible edge effect. This concludes the proof. 
Lemma 4.11. Let $N$ be a stationary point process under $P$ with intensity $\lambda$ and let $P^{0}$ denote the Palm probability associated to $P$. Let $\gamma>0$. Assume that there exist $\delta \in(0,1)$ and $q>0$ such that

$$
\sup _{k \geq 1} k^{-q \delta} \mathbb{E}^{0}\left[\left|t_{k}-\lambda^{-1} k\right|^{q}\right]<\infty
$$

If (4.26) holds with $q \geq \gamma+1$, then

$$
\sup _{t \geq 2} \mathbb{E}\left[\left(\frac{N_{i}(t)}{t}\right)^{-\gamma} \mathbf{1}_{\left\{N_{i}(t)>0\right\}}\right]<\infty .
$$

If (4.26) holds with $q>1+\gamma /(1-\delta)$, then $\mathbb{E}\left[N^{\gamma}(1)\right]<\infty$.

Proof. For $k \geq 2$, define $c_{k}=(k-1)^{-\gamma}-k^{-\gamma}$. Then, $\sum_{k=2}^{\infty} c_{k}=1$ and applying summation by parts, we have

$$
\begin{aligned}
\mathbb{E}\left[N^{-\gamma}(t) \mathbf{1}_{\{N(t)>0\}}\right] & =\sum_{k=1}^{\infty} k^{-\gamma} \mathbb{P}(N(t)=k)=\sum_{k=1}^{\infty} k^{-\gamma}\{\mathbb{P}(N(t) \geq k)-\mathbb{P}(N(t) \geq k+1)\} \\
& =\mathbb{P}(N(t) \geq 1)-\sum_{k=2}^{\infty} c_{k} \mathbb{P}(N(t) \geq k) \\
& =\mathbb{P}\left(t_{1} \leq t\right)-\sum_{k=2}^{\infty} c_{k} \mathbb{P}\left(t_{k} \leq t\right)=-\mathbb{P}\left(t_{1}>t\right)+\sum_{k=2}^{\infty} c_{k} \mathbb{P}\left(t_{k}>t\right) .
\end{aligned}
$$

Without loss of generality, assume that the intensity of the point process is $\lambda=1$. Then, by definition of $c_{k}$, we have, for $t \geq 2$,

$$
t^{\gamma} \sum_{k \geq[t / 2]+1} c_{k} \mathbb{P}\left(t_{k}>t\right) \leq t^{\gamma}([t / 2])^{-\gamma}=O(1) .
$$

For $k \leq[t / 2]$, we have, by Markov's inequality,

$$
\mathbb{P}\left(t_{k}>t\right)=\mathbb{P}\left(t_{k}-k>t-k\right) \leq \mathbb{P}\left(t_{k}-k>t / 2\right) \leq c t^{-\gamma} \mathbb{E}\left[\left|t_{k}-k\right|^{\gamma}\right]
$$

Applying the Ryll-Nardzewski inversion formula (Baccelli and Brémaud (2003, Formula 1.2.25)), we have

$$
\mathbb{E}\left[\left|t_{k}-k\right|^{\gamma}\right]=\mathbb{E}^{0}\left[t_{1}\left|t_{k}-k\right|^{\gamma}\right] \leq\left\{\mathbb{E}^{0}\left[t_{1}^{1+\gamma}\right]\right\}^{1 /(\gamma+1)}\left\{\mathbb{E}^{0}\left[\left|t_{k}-k\right|^{\gamma+1} \mid\right]\right\}^{\gamma /(\gamma+1)} .
$$

Thus, applying Condition (4.26), we obtain that $\mathbb{P}\left(t_{k}>t\right) \leq c^{\prime} t^{-\gamma} k^{\gamma \delta}$ and thus

$$
t^{\gamma} \sum_{2 \leq k \leq[t / 2]} c_{k} \mathbb{P}\left(t_{k}>t\right) \leq c^{\prime} \sum_{2 \leq k \leq[t / 2]} c_{k} k^{\gamma \delta} \leq c^{\prime} \sum_{2 \leq k \leq[t / 2]} k^{-\gamma(1-\delta)-1}=O(1) .
$$


This concludes the proof of (4.27). We now consider the positive moments of $N(1)$. Applying summation by part, we have

$$
\mathbb{E}\left[N^{\gamma}(1)\right]=\sum_{k=1}^{\infty}\left\{k^{\gamma}-(k-1)^{\gamma}\right\} \mathbb{P}(N(1) \geq k)=\sum_{k=1}^{\infty}\left\{k^{\gamma}-(k-1)^{\gamma}\right\} \mathbb{P}\left(t_{k} \leq 1\right) .
$$

For $k \geq 2$ and $q>0$, we have, still assuming that $\lambda=1$,

$$
\mathbb{P}\left(t_{k} \leq 1\right) \leq \mathbb{P}\left(t_{k}-k \leq-k / 2\right) \leq \mathbb{E}\left[\left|t_{k}-k\right|^{q}\right] k^{-q}
$$

Applying again the Ryll-Narzewski formula and Condition (4.26), we obtain, for $k \geq 2$,

$$
\mathbb{P}\left(t_{k} \leq 1\right) \leq c k^{-q(1-\delta)}
$$

Thus,

$$
\mathbb{E}\left[N^{\gamma}(1)\right] \leq 1+c \sum_{k=1}^{\infty}\left\{k^{\gamma}-(k-1)^{\gamma}\right\} k^{-q(1-\delta)}
$$

The series is convergent as long as $q(1-\delta)>\gamma$.

Proof of (3.4) for the LMSD model. Consider the LMSD model of Example 2.1. It is proved in Deo et al. (2009a, Proposition 1) that (4.26) holds with $\delta=H_{\tau}$ if $\mathbb{E}\left[\epsilon_{0}^{p}\right]<\infty$ for all $p \geq 1$. Actually, a close inspection of the first lines of the proof shows that only $q$ finite moments of $\epsilon_{0}$ are needed. Thus (3.4) holds if $E^{0}\left[\epsilon_{0}^{9-4 H}\right]<\infty$, and $\mathbb{E}\left[N^{4}(1)\right]<\infty$ if $E^{0}\left[\epsilon_{0}^{q}\right]<\infty$ for some $q>1+4 /\left(1-H_{\tau}\right)$.

Proof of (3.4) for the ACD model. Under the assumptions of Example 2.2, the sequence $\left\{\tau_{k}\right\}$ is geometrically $\beta$-mixing Carrasco and Chen (2002, Proposition 17). Denote $m=$ $\mathbb{E}^{0}\left[\tau_{1}\right]$. The sequence $\left\{t_{k}\right\}$ is geometrically mixing, hence geometrically strong mixing. Thus, by Rio (2000, Theorem 2.5), for $q \geq 2$, if $E^{0}\left[\tau_{1}^{q+1+\epsilon}\right]<\infty$ for some $\epsilon>0$, then $E^{0}\left[\left|t_{n}-m n\right|^{q+1}\right]=O\left(n^{(q+1) / 2}\right)$. Thus (4.26) holds with $\delta=1 / 2$.

\section{References}

Miguel A. Arcones. Limit theorems for nonlinear functionals of a stationary Gaussian sequence of vectors. The Annals of Probability, 22(4):2242-2274, 1994.

François Baccelli and Pierre Brémaud. Elements of queueing theory, volume 26 of Applications of Mathematics (New York). Springer-Verlag, Berlin, second edition, 2003.

Ole E. Barndorff-Nielsen, Peter Reinhard Hansen, Asger Lunde, and Neil Shephard. Designing realized kernels to measure the ex post variation of equity prices in the presence of noise. Econometrica, 76(6):1481-1536, 2008. 
Luc Bauwens and David Veredas. The stochastic conditional duration model: a latent variable model for the analysis of financial durations. Journal of Econometrics, 119(2): 381-412, 2004. Dynamic factor models.

Patrick Billingsley. Convergence of probability measures. John Wiley \& Sons Inc., New York, 1968.

Marine Carrasco and Xiaohong Chen. Mixing and moment properties of various GARCH and stochastic volatility models. Econometric Theory, 18(1):17-39, 2002.

Willa W. Chen and Clifford M. Hurvich. Estimating fractional cointegration in the presence of polynomial trends. Journal of Econometrics, 117(1):95-121, 2003a.

Willa W. Chen and Clifford M. Hurvich. Semiparametric estimation of multivariate fractional cointegration. Journal of the American Statistical Association, 98(463):629-642, 2003b.

Daryl J. Daley and David Vere-Jones. An introduction to the theory of point processes. Vol. I: Elementary theory and methods. 2nd ed. Probability and Its Applications. New York, NY: Springer., 2003.

Rohit Deo, Clifford Hurvich, and Yi Lu. Forecasting realized volatility using a long-memory stochastic volatility model: estimation, prediction and seasonal adjustment. Journal of Econometrics, 131(1-2):29-58, 2006.

Rohit Deo, Clifford M. Hurvich, Philippe Soulier, and Yi Wang. Conditions for the propagation of memory parameter from durations to counts and realized volatility. Econometric Theory, 25(3):764-792, 2009a.

Rohit Deo, Clifford M. Hurvich, Philippe Soulier, and Yi Wang. Conditions for the propagation of memory parameter from durations to counts and realized volatility. Econometric Theory, 25(3):764-792, 2009b.

Rohit Deo, Mengchen Hsieh, and Clifford M. Hurvich. Long memory in intertrade durations, counts and realized volatility of nyse stocks. Journal of Statistical Planning and Inference, 140(12):3715 - 3733, 2010. doi: DOI:10.1016/j.jspi.2010.04.037. Special Issue in Honor of Emanuel Parzen on the Occasion of his 80th Birthday and Retirement from the Department of Statistics, Texas A\&M University - Emmanuel Parzen.

Robert F. Engle and Jeffrey R. Russell. Autoregressive conditional duration: a new model for irregularly spaced transaction data. Econometrica, 66(5):1127-1162, 1998.

Eric Ghysels, Pedro Santa-Clara, and Rossen Valkanov. Predicting volatility: getting the most out of return data sampled at different frequencies. Journal of Econometrics, 131 (1-2):59-95, 2006. 
Liudas Giraitis, Peter M. Robinson, and Donatas Surgailis. Variance-type estimation of long memory. Stochastic Process. Appl., 80(1):1-24, 1999.

P. Hall and C. C. Heyde. Martingale limit theory and its application. Academic Press Inc. [Harcourt Brace Jovanovich Publishers], New York, 1980. Probability and Mathematical Statistics.

James D. Hamilton. Time series analysis. Princeton University Press, Princeton, NJ, 1994.

Clifford M. Hurvich and Yi Wang. A pure-jump transaction-level price model yielding cointegration, leverage, and nonsynchronous trading effects. Working Paper, Stern School of Business, New York University, 2009.

Clifford M. Hurvich and Yi Wang. A pure-jump transaction-level price model yielding cointegration. Journal of Business 65 Economic Statistics, 28(4):539-558, 2010.

Ildar Abdulovich Ibragimov and Yuri Anatolevich Rozanov. Gaussian random processes, volume 9 of Applications of Mathematics. Springer-Verlag, New York, 1978. Translated from the Russian by A. B. Aries.

Domenico Marinucci and Peter M. Robinson. Weak convergence of multivariate fractional processes. Stochastic Processes and their Applications, 86(1):103-120, 2000.

Jean-Luc Prigent. Option pricing with a general marked point process. Mathematics of Operations Research, 26(1):50-66, 2001.

Sidney I. Resnick. Extreme values, regular variation, and point processes, volume 4 of Applied Probability. A Series of the Applied Probability Trust. Springer-Verlag, New York, 1987.

Sidney I. Resnick. Heavy-tail phenomena. Springer Series in Operations Research and Financial Engineering. Springer, New York, 2007. Probabilistic and statistical modeling.

Emmanuel Rio. Théorie asymptotique des processus aléatoires faiblement dépendants, volume 31 of Mathématiques $\&$ Applications (Berlin) [Mathematics $\&$ Applications]. Springer-Verlag, Berlin, 2000.

Peter M. Robinson and Domenico Marinucci. Narrow-band analysis of nonstationary processes. The Annals of Statistics, 29(4):947-986, 2001.

Wim Vervaat. Functional central limit theorems for processes with positive drift and their inverses. Z. Wahrscheinlichkeitstheorie und Verw. Gebiete, 23:245-253, 1972.

Ward Whitt. Stochastic-process limits. Springer Series in Operations Research. SpringerVerlag, New York, 2002. An introduction to stochastic-process limits and their application to queues. 